\title{
The transmembrane protein Opy2 mediates activation of the Cek1 MAP kinase in Candida albicans
}

Carmen Herrero de Dios, Elvira Román, Carla Diez, Rebeca Alonso-Monge*and Jesús Pla.

Departamento de Microbiología II. Facultad de Farmacia. Universidad Complutense de Madrid.

Plaza de Ramón y Cajal s/n. E-28040 MADRID. SPAIN

Phone: + 34913941617

FAX: $\quad+34913941745$

*Corresponding author: realonso@farm.ucm.es

Keywords

SVG, osmotic stress, cell wall integrity, pathogenic fungus, MAPK phosphorylation, Candida albicans

Abbreviations

MAPK, Mitogen Activated Protein Kinase; SVG, Sterile Vegetative Growth 


\section{Abstract}

MAPK pathways are conserved and complex mechanisms of signaling in eukaryotic cells. These pathways mediate adaptation to different stress conditions by a core kinase cascade that perceives changes in the environment by different upstream elements and mediates adaptation through transcription factors. In the present work, the transmembrane protein Opy2 has been identified and functionally characterized in Candida albicans. This protein is required to trigger Cek1 phosphorylation by different stimuli such as the resumption of growth from stationary phase or the addition of the cell wall disturbing compounds zymolyase and tunicamycin. opy2 mutants display susceptibility to cell wall disturbing compounds like Congo red. However, it does not play a role in the adaptation to high osmolarity or oxidative stress, in close contrast with the situation for the homolog protein in S. cerevisiae. The overexpression of Opy2 in a S. cerevisiae opy2 ssk1 mutant partially complemented the osmosensitivity on solid medium by a Hog1-independent mechanism as well as alleviated the abnormal morphology observed in this mutant under high osmolarity. The electrophoretic pattern of CaOpy2 tagged version in S. cerevisiae suggested similar post-translational modification in both microorganisms. This protein is also involved in pathogenesis as revealed by the fact that opy2 mutants displayed a significantly reduced virulence in the Galleria mellonella model. 


\section{Introduction}

Cells respond and adapt to external changes through signal transduction pathways. Among the different mechanisms of cell signaling described in eukaryotic cells the MAPK (Mitogen Activated Protein Kinase) mediated pathways are essential. These signaling routes are conserved in all eukaryotic cells and are implicated in several relevant physiological functions for the cell (Kultz, 1998). MAPK pathways consist on a module of three kinases (a MAP kinase kinase kinase, a MAP kinase kinase and a MAP kinase) that are activated by successive phosphorylation in response to different signals through others kinases, two-component systems and/or heterotrimeric $\mathrm{G}$ proteins.

Candida albicans is a human commensal that is able to cause infection under certain circumstances and therefore, it is consider as an opportunistic pathogen (Odds, 1988). These infections range from superficial to systemic which are frequently difficult to treat. Although others species of Candida have increased their prevalence as nosocomial systemic infections, C. albicans still remains as the major cause of fungemia in hospitals of developed countries. In C. albicans four MAP kinases have been currently identified: $\mathrm{Mkc1}$, the homolog to S. cerevisiae S1t2 (Navarro-García et al., 1995); Cek1, homolog to Kss1 (Csank et al., 1998); Cek2, homolog to Fus3 (Chen et al., 2002) and Hog1, homolog to the Hog1 MAP kinase (San José et al., 1996). Mkc1 participates in the cell wall integrity pathway and is implicated in cell wall biogenesis, the response to cell wall stress and others like biofilm formation and virulence (Diez-Orejas et al., 1997; Kumamoto, 2005; Navarro-Garcia et al., 2005b; Navarro-García et al., 1995; Navarro-García et al., 1998). Cek2 participates in mating as cek1cek2 double mutants are unable to mate. Cek1 is involved in invasive hyphal growth, mating efficiency, cell wall construction, quorum sensing and virulence in a systemic murine model (Chen et al., 2002; Csank et al., 1998; Roman et al., 2009a; Roman et al., 2005; Roman et al., 2009b). Finally, theHog1 kinase responds to different stresses such as oxidative, osmotic and the presence of 
metals, etc. (Alonso-Monge et al., 2003; Smith et al., 2004). This kinase is also implicated in the yeast-to-hypha transition, cell wall biogenesis, oxidative metabolism and virulence (AlonsoMonge et al., 1999; Alonso-Monge et al., 2009; San José et al., 1996).

In S. cerevisiae, Hog1 perceives the signal via two branches: the Msb2, Sho1, Ste50, Ste11 branch and the Sln1-Ypd1-Ssk1, Ssk2/Ssk22 branch (O'Rourke and Herskowitz, 2002; Posas et al., 1996). In addition, another transmembrane mucin Hkr1 has been reported as potential osmosensor of the Sho1 branch. Hkr1 and Msb2 act upstream Sho1 forming a complex which, upon high external osmolarity, triggers Shol mediated signaling (Tatebayashi et al., 2007). Opy2 is a transmembrane protein first postulated as a third osmosensor in the Sho1 branch that anchors Ste50 and Ste11 (Wu et al., 2006). The association between Ste50 and Opy2 is regulated positively by external nutritional conditions or negatively by activated MAP kinases. This association/dissociation controls signal transmission integrating different signals and MAPK network regulation (Yamamoto et al., 2010).

In C. albicans the analysis of MAPK pathways were performed originally with the MAP Kinases, actually, others elements have been included to draft the structure and organization of these pathways. Initially two branches were allocated in the HOG pathway leading to Pbs2 phosphorylation which is the Hog1 MAPKK (Arana et al., 2005). Nevertheless it seems that only the branch involving Sln1, Ypd1, Ssk1 and Skk2 plays an unequivocal role in the Pbs2Hog1 signaling (Cheetham et al., 2007). The second via, integrated by Sho1 and Ste11 triggers Cek1 phosphorylation; these elements belong to the so-called SVG (Sterile Vegetative Growth) pathway (Lee and Elion, 1999; Román et al., 2005). Also belonging to this pathway, the Msb2 mucin mediates Cek1 upon tunicamycin addition (Román et al., 2009b), although the hierarchy among the transmembrane elements (Sho1 and Msb2) of the SVG pathway has not yet been analyzed. 
Although Hog 1 phosphorylation seems to be mediated by Ssk1 and Ssk2 in C.albicans, deletion of SSK1 in addition to MSB2 and SHO1 genes rendered cells that are still able to activate Hog1 upon osmotic stress (Roman et al., 2009b). The triple mutant ssk1 sho1 msb2 displayed an aberrant cell morphology under hypertonic conditions in spite of accumulating intracellular glycerol and Hog1 translocation to the nucleus (Roman et al., 2009b). These results suggest that there may be present others elements still unknown implicated in the phosphorylation of Hog1 under osmotic stress. In order to identify this/these elements, the OPY2 gene was deleted in C. albicans and its function was analyzed both in the SVG and the HOG pathways. Our results demonstrate that the transmembrane protein Opy2 mediates Cek1 phosphorylation upon the presence of cell wall disturbing agents and during the resumption from stationary phase of growth. The opy 2 single mutant displayed cell wall defects while it plays no apparent role on the susceptibility to osmotic or oxidative stress. We also evidence functional differences between C. albicans and S. cerevisiae regarding signaling evolution.

\section{Material and methods}

\section{Strains and growth conditions}

Yeast strains are listed in Table 1. For clarity, and unless otherwise stated, opy2 will always indicate the homozygous C. albicans mutant opy2/opy2strain (strain CHO4-1), opy $2^{\text {reint }}$ designates the homozygous opy2/opy2where the OPY2 gene was reintegrated at the OPY2 region under the control of its own promoter (strain CHO39).

C. albicans strains were grown in YPD medium ( $1 \%$ yeast extract, $2 \%$ peptone, $2 \%$ glucose) at $37^{\circ} \mathrm{C}$, unless indicated. Usually, overnight cultures were refreshed to an optical density of 0.1 (measured at $600 \mathrm{~nm}$ ) and experiments were performed when cultures reached an optical density of 1 (at $\lambda 600 \mathrm{~nm}$ ) when exponential phase cells were required. In the case of stationary 
phase cells, cells from a 1 day overnight culture were routinely used. The cultures of strains carrying the $O P Y 2-m y c$ under the control of tetracycline inducible promoter were incubated in YPD supplemented with $10 \mu \mathrm{g} / \mathrm{ml}$ doxicycline for one day to ensure OPY2-myc full expression. S. cerevisiae strains were grown routinely in minimal medium ( $2 \%$ glucose, $0.67 \%$ yeast nitrogen base without aminoacids) supplemented with a synthetic complete URA- drop-out to impede loss of plasmid. Cultures were incubated at $30^{\circ} \mathrm{C}$, unless otherwise indicated.

Sensitivity on solid media was tested on YPD supplemented with the indicated concentration of several compounds (hydrogen peroxide, menadione, $\mathrm{NaCl}$, sorbitol, Congo red or calcofluor white). In the case of strains carrying the inducible version of tetracycline promoter $\left(\mathrm{TET}^{\mathrm{P}}\right)$, doxicycline was added to the plates at $20 \mu \mathrm{g} / \mathrm{ml} .10^{5}, 10^{4}, 10^{3}$ and $10^{2}$ cells were spotted on the plates and incubated unless otherwise stated at $37 \mathrm{C}$ to reveal differences of growth. In the case of S. cerevisiae strains, the temperature of incubation was $30^{\circ} \mathrm{C}$.

The assays in liquid medium to visualize cell morphology were performed as follow. Cells were grown overnight in YPD or YPD supplemented with $1 \mathrm{M} \mathrm{NaCl}$ and visualized with a Nikon TE2000 fluorescence inverted microscope equipped with CCD (Melville, NY). Digital images were acquired with an Orca C4742-95-12ER camera (Hamamatsu, Bridgewater, NJ) and processed with the HCImage software.

\section{Genetic constructions}

For the disruption of the OPY2 gene, we constructed the pDOPY2 plasmid. A $0.72 \mathrm{kbp}$ ORF flanking region was amplified by PCR using the primers OPY2KpnI and OPY2XhoI. This fragment was first subcloned in the commercial vector pGEM-T (Promega). Similarly, oligonucleotides OPY2NotI and OPY2SacI were employed to amplify a $0.529 \mathrm{kbp}$ region flanking the 3' of the OPY2 gene of the CAF2 strain and cloned in pGEM-T vector. Both 
fragments were finally accommodated in the pSF2SA plasmid (Reuss et al., 2004) carrying the nourseothricin resistance marker (CaSAT1), thus obtaining pDOPY2. This plasmid was digested with KpnI-SacI to force the homologous recombination at the OPY2 chromosomal region. Following the already described SAT1 flipping method (Reuss et al., 2004), two rounds of integration/excision were necessary to generate homozygous mutants.

To construct the OPY2-myc fusion we previously generated the pNIM1_MoGFP_carboxi_ca_myc plasmid which derives from the pNIM1 vector (Park and Morschhauser, 2005). The primers: ETC-myc-upper: 5'-CAGTTTGGTTCAGCACCTTGTCG3' and ETC-myc-lower: 5'-GCCAGATCTTTACAAGTCTTCTTCAGAAATCAAT TTTTGTTCGCGGCCGCATTTGTATAGTTCATCCATGCCATGTG-3') allowed to amplify the GFP fused to a myc tag from the pNIM1 vector. The PCR product was digested with SalI and BglII, allowing the placement in the SalI-BglII places again in the pNIM1 plasmid. We then amplified the OPY2 ORF by PCR using the primers o-OPY2GFPmycUp (5'GCGGTCGACAATCATGCCAATACCCAGATC) and o-OPY2GFPmycLow (5'GCAGCGGCCGCCGCTCGAGAGTTTGTCAGGATCAGCAAAAG-3') and genomic DNA form CAF2 strain as template. For the pNIM1-Opy2-myc construction, where the Opy2-myc fusion is under the control of the inducible tetracycline promoter, the PCR product (1615 bp) was cloned in the SalI-NotI sites of pNIM1_MoGFP_carboxi_ca_myc after digestion with the same enzymes. The construction was integrated in the ADH1 locus after digestion with KpnISacII.

The opy2 reintegrant strain was constructed using a vector that allowed the fusion of the gene to HA and the plasmid integration in the ARD1 locus (constructed and kindly provided by Daniel Prieto). The OPY2 ORF plus $1.5 \mathrm{~Kb}$ upstream of the ATG was amplified using the primers: prOPY2opy2UP (CGGTCGACGAAAAGAAATCCCAATGACGTAGTGGTAGTG) and prOPY2opy2LOW (GCGGATCCTTTGTCAGGATCAGCAAAAGGGCTTC). After digestion 
with SalI-BamHI, the amplified sequence (3168 bp) was located in the mentioned plasmid. The final construction was integrated in the OPY2 own promoter and in the ARD1locus (after digestion with AccI or KpnI-SacI, respectively). The correct integration was checked by PCR using the primers: IntgprOPY2UP: 5'-CAGATTTTCCAATGAGAGAGCCGG-3' and IntgprOPY2Low: 5'-GCGGATCCTTTGTCAGGATCAGCAAAAGGGCTTC-3' and functional complementation.

\section{Genetic constructions for $S$. cerevisiae and transformation}

To amplify the CaOPY2-myc fusion the previously obtained plasmidpDOPY2 was used as DNA template. The primers utilized were: o-CaOpy2Bup (CCGGATCCAATCAATCATGCCAATACCCAGATCG) as the forward primer and the oCaOpy2Plw (GGCTGCAGTTACAAGTCTTCTTCAGAAATCAATTTTTTGTTC) as the reverse one. The PCR product was inserted into the pGEM-T commercial vector (Promega) obtaining the CaOPY2myc-pGEM-T plasmid. This plasmid was digested with BamHI-PstI to accommodate the fusion CaOPY2-myc into the BamHI and PstI sites of the pCM189 vector (Gari et al., 1997) to generate the CaOPy2-myc-pCM189 plasmid. The ScOPY2 ORF was amplified using S. cerevisiae genomic DNA as template and the primers o-SCOPY2up (CCGGATCCATGTCGAGCTCCTC) and o-ScOPY2lw (CCCGCGGCCGCCTCGTTCATCG). The PCR product of $1097 \mathrm{pb}$ was inserted in the pGEM-T commercial vector prior the digestion with BamHI-NotI. The CaOPy2-myc-pCM189 plasmid was digested with BamHI-NotI and the CaOPY ORF was substituted for the ScOPY2 ORF allowing the fusion to the myc tag. The resulting plasmid was named as ScOPY2-mycpCM189. To introduce the generated plasmids into S. cerevisiae, the one-step transformation protocol was used (Chen et al., 1992). 


\section{Protein extracts and immunoblot analysis}

In the case of C. albicans, strains were grown to an optical density of 1 at $37^{\circ} \mathrm{C}$ in YPD. $1 \mathrm{M}$ $\mathrm{NaCl}$ or $10 \mathrm{mM}$ hydrogen peroxide was added to the medium at the final concentration indicated and samples were taken at different time points as indicated in the figures. Alternatively, cells in exponential phase (1 O.D.) were challenged with $20 \mu \mathrm{g} / \mathrm{ml}$ Congo red (CR), $2 \mathrm{U} / \mathrm{ml} \mathrm{100-T} \mathrm{zymoliase} \mathrm{(Z)} \mathrm{or} 2.5 \mu \mathrm{g} / \mathrm{ml}$ tunicamycin ( $\mathrm{Tn}$ ) and incubated for $1 \mathrm{~h}$ at $37^{\circ} \mathrm{C}$. The resumption of growth experiments were performed with overnight cultures growth in YPD at $37^{\circ} \mathrm{C}$ and refreshed in pre-warmed YPD medium, sample were collected after 30 minutes or 1h of incubation.

The response to osmotic stress in S. cerevisiae was performed growing cells in minimal medium and $30^{\circ} \mathrm{C}$. Overnight cultures were diluted to 0.2 O.D. and incubated for $5 \mathrm{~h}$ approximately. At this point, $\mathrm{NaCl}$ was added to the medium $(0.9 \mathrm{M})$ and samples were collected at different time points. Stationary phase cultures were used for detection of the myc fusion,

For both microorganisms, cell extracts were obtained as previously indicated (Martin et al., 2000). Even amounts of proteins were loaded onto gels as assessed by $280 \mathrm{~nm}$ measurement of the samples and Ponceau Red staining of the membranes prior to blocking and detection. Blots were probed with phospho-p38 MAP kinase (Thr180/Tyr182) 3D7 monoclonal antibody (Cell Signaling Technology, Inc.), ScHog1 polyclonal antibody (Santa Cruz Biotechnology), phospho-p44/42 MAP kinase (Thr202/Tyr204) (D13.14.4E)(Cell Signaling Technology, Inc.), anti-myc Tag, clone 4A6 (Millipore), Mouse anti-actin, clone C4 (MP Biomedicals) and Ab-Cek1 antibodies (Román et al., 2005) or Ab-Mkc1 (Navarro-Garcia et al., 2005). The detection was performed using the Quantitative Fluorescent Imaging System Odissey from Licor. 


\section{Virulence assays in Galleria mellonella}

G. mellonella in their larvae form were infected with $10^{6} \mathrm{CFUsper}$ larvae with C. albicans cells. The strains CAF2, opy2 and OPY2reintegrant grown over night at $37^{\circ} \mathrm{C}$ were washed twice with PBS, the number of cell was quantified using a Neubauer chamber and the inoculum $\left(10^{6}\right.$ cells $)$ was injected directly into the hemocoel at the last left pro-leg with a Hamilton syringe. Each infection group contained 20 larvae of approximately 400-500 mg size. Two additional controls (without inoculation and with PBS injection) were included. Survival at $37^{\circ} \mathrm{C}$ was monitored for 6 days after infection. Kaplan-Meier survival curves are shown.

\section{Mammalian cell culture and Candida albicans infections assays}

The HL-60 cell line was obtained from ATCC (American Type Culture Collection) and was maintained in RPMI 1640 (Gibco) supplemented with 10\% heat inactivated fetal bovine serum (Gibco), glutamine (Gibco) and 1\% streptomycin/penicillin (Gibco) at $37^{\circ} \mathrm{C}$ in $5 \% \mathrm{CO}_{2}$. HL-60 cells were differentiated by incubation in presence of DMSO (dimethyl sulfoxide) (Merck) $1.3 \%(\mathrm{v} / \mathrm{v})$ for 4 days. For harvesting, cells were centrifuged at 1000 r.p.m, washed with PBS and resuspended in RPMI at the required final concentration. The cell population was counted by trypan blue dye exclusion in a hemocytometer.

Killing assays were performed in 24 -well tissue culture dishes. Yeasts strains were grown in YPD overnight at $37^{\circ} \mathrm{C}$, washed twice with phosphate-buffered saline (PBS), counted and resuspended in RPMI 1640 medium. Candida and neutrophil-like cells were mixed at a cell ratio of 1:40 (yeasts: neutrophils). The cultures were incubatedat $37^{\circ} \mathrm{C}$ in $5 \% \mathrm{CO}_{2}$ for 2 hours. At the end of this period, $0.05 \%$ SDS in water was added to lyse the neutrophils, and serial dilutions of all strains were performed. $100 \mu 1$ of each dilution were spread onto YPD plates and 
determinations of colony forming units (CFUs) were performed after incubation for 24 hours at $37^{\circ} \mathrm{C}$.

The assays were done a total of four times, and viability of each strain was expressed as the percentage of CFUs from phagocyte-yeasts co-cultures versus simultaneous cultures containing C. albicans without phagocytes according to the formula [100-(CFUs without phagocytes) (CFUs with phagocytes) / (CFUs without phagocytes)] X 100.

\section{Results}

\section{OPY2 encodes a transmembrane protein involved in cell wall}

\section{biogenesis}

A predicted ortolog to S. cerevisiae OPY2 gene was identified in chromosome 1 of C. albicans. The putative CaOPY2 ORF (other alias orf19.12576, IPF22134.1, IPF13257.2) is 1584 bp long and encodes a 528 -amino-acid protein sharing $37 \%$ of identity with S. cerevisiae Opy 2 . In this organism, OPY2 encodes a significant smaller protein of 361 aminoacids with a transmembrane domain between aminoacids 93 to 115 (Ekiel et al., 2009). CaOPY2 also displays a transmembrane region from aminoacids72 to 94 as predicted by the SMART software (http://smart.embl-heidelberg.de/) (Fig. 7A).

In order to analyze the function of Opy2 in C. albicans we deleted the OPY2 gene following the strategy described by Reuss and co-workers (Reuss et al., 2004) based on the SAT1 gene which confers resistance to nourseotricine. The deletion was performed in the CAF2 strain as well as in mutants defective in different elements of the SVG and/or HOG pathways (Table 1) in order to establish epistatic relationships among them. 
The susceptibility to compounds that interfere with cell wall biogenesis was analyzed. The deletion of OPY2 rendered cells almost as sensitive to Congo red as those displayed by cek1 mutants (Fig. 1A). When assayed at $42^{\circ} \mathrm{C}$ both opy2 and cek1 mutants behaved similar. The reintegration of OPY2 gene either under the control of its own promoter or under the tetracycline inducible promoter reverted this phenotype indicating that the lack of Opy2 is the responsible for this phenotype and that both OPY2versions were functional. Both sho1 and $m s b 2$ have been described to result in enhanced sensitivity to Congo red (Roman et al., 2005; Roman et al., 2009b; Roman et al., 2009a). Deletion of OPY2 in these backgrounds did not significantly increase the susceptibility to Congo red of the single mutants (Fig. 1B), suggesting a linear relationship between both Opy2 and Sho1/Msb2. Remarkably, deletion of OPY2 did not render cells susceptible to caspofungin or tunicamycin on solid media, in sharp contrast with the phenotype displayed by sho1 or cek1 (Supplementary Fig.1).

\section{Opy2 is required for Cek1 phosporylation}

As previously described, Cek1 becomes phosphorylated in response to different environmental changes such as the addition of cell wall disturbing agents or during the resumption from stationary phase when cells are diluted in fresh medium (Roman et al., 2005). Given the phenotypic similarities between cek1 and opy2 mutants, we tested the role of Opy2 in controlling Cek1 activation and analyzed the pattern of Cek1 phosphorylation in the presence of tunicamycin, zymolyase, Congo red and under the resumption of growth when the culture was diluted in pre-warmed fresh medium (Fig. 2A and 2B). These conditions have been previously shown to be required for Cek1 activation. Opy2 was necessary for the activation of Cek1 in all these circumstances and this effect was dependent of the presence of a functional Opy2 gene as shown by revertant strains. 
As it has been previously described, certain mutants in the HOG pathway hyperphosphorylate Cek1 in a Sho1-dependent manner (Roman et al., 2005) by a crosstalk mechanism. We further studied whether this was also dependent on Opy2 by constructing double ssk1 opy2 mutants. As shown in Figure 2C, the increased phosphorylation of Cek1 detected in ssk1 mutants was completely blocked when the OPY2 gene was deleted. In addition, the lack of Opy2 also increased the susceptibility to Congo red in an ssk1 mutant to levels similar to those observed in opy2 mutants (Fig. 2C). This result is in agreement with the hypothesis that the resistance to Congo red observed in HOG pathway mutants is due to high Cek1 phosphorylation levels (Eisman et al., 2006).

\section{Opy2 has no role in the response to osmotic and oxidative stress}

The role of Opy 2 protein in the HOG pathway was analyzed by solid media susceptibility assays. These studies showed that the deletion of Opy2 did not impair the growth neither on high osmotic media nor in the presence of oxidative agents when compared to parental strains (Fig. 3 and supplementary 2). Single deletions of Sho1, Msb2 or Opy2 have no significant effect on the osmosensitivity, neither as single mutants nor in combination of two or three elements (Sho1, Msb2 and Opy2) (Data not shown). However, when the response regulator of the twocomponent system Ssk1 is absent in combination with the lack of Sho1 or Opy2 the sensitivity to osmotic stress increased appreciably (Fig. 3A). This osmosensitivity was comparable to the osmosensitivity displayed by triple ssk1 msb2 sho1and quadruple ssk1 msb2 sho1 opy2 mutants and to the hog1 mutant.

The deletion of OPY2 gene did not significantly alter the phosphorylation pattern of Hog1 upon addition of $1 \mathrm{M} \mathrm{NaCl}$ since the opy2 mutant followed a similar kinetic to wild type strain (Fig. 3B). However, an increased basal Hog1 phosphorylation could be detected in opy2 exponentially growing cells. Moreover, no differences were detected regarding the intracellular 
glycerol accumulation and STL1 expression (a target of the HOG pathway in C. albicans) in opy2 mutants compared to wild type (data not shown). These results indicate that the response via Hog1 to osmotic stress is not significantly altered when Opy2 is absent. Nevertheless, deletion of the OPY2 gene in a ssk1 background leaded to an increased osmosensitivity (Fig. 3A), this phenotype did not correlate with an impaired Hog 1 phosphorylation given that the ssk1 opy2 double mutant was able to activate Hog1 similarly to wild type strain.

In the case of oxidative stress, the lack of Ssk1 rendered sensitivity to oxidative agents such as menadione or hydrogen peroxide and impaired Hog1 phosphorylation upon hydrogen peroxide addition (Chauhan et al., 2003). However, no effect was observed when others elements (Sho1, Msb2 and/or Opy2) were absent suggesting that only the Ssk1-mediated pathway plays a significant role in oxidative stress response ( Supplementary Fig. 2) (Cheetham et al., 2007; Roman et al., 2005).

Taken these results together we conclude that the main branch involved in Hog1 signaling is mediated by Ssk1 as it has been previously shown (Chauhan et al., 2003; Cheetham et al., 2007). This role is crucial upon oxidative stress; nevertheless, regarding adaptation to osmotic stress, both branches may play a role.

\section{Opy2 is not involved in the signaling to Hog1 in S. cerevisiae}

Some differences can be detected at the sequence level between S. cerevisiae and C. albicans Opy2 proteins. In S. cerevisiae Opy2 binds Ste50 through a conserved region (CR)-B sited in its cytoplasmic tail; this interaction leads to Hog1 phosphorylation in response to osmotic stress. This domain is, however, absent in theCaOpy2 (Fig. 7A). In order to determine if this protein can play a role within the HOG pathway in S. cerevisiae, both CaOPY2 and ScOPY2 genes were myc-tagged and introduced in the TET-dependent expression vector pCM189 from 
S. cerevisiae. The plasmids obtained were introduced both in a wild type and a opy2 ssk1 mutant (Wu et al., 2006). CaOpy2 was only partially able to complement the osmosensitivity of the S. cerevisiae opy2 ssk1 mutant (Fig. 4A) while, as expected, ScOPY2 completely complement the osmosensitive phenotype. Interestingly, the S. cerevisiae opy2 ssk1mutant became osmotolerant when grown at $37^{\circ} \mathrm{C}$ (Data not shown). This behavior correlates with mutant defective in the HOG pathway but no with ste11 mutant which remain osmosensitive at $37^{\circ} \mathrm{C}$ probably due to its belonging to both parhways, SVG and HOG (Alonso-Monge et al., 2001).

The opy2 ssk1 mutant exhibited altered cell morphology when growing under hyperosmotic liquid medium, showing elongated or enlarged cells. These morphologies were not observed when the ScOPY2 gene was introduced and the expression of the homologous C. albicans gene partially complemented the altered morphology (Fig.4B).

In parallel, the ability to activate the HOG pathway was analyzed upon an osmotic challenge. Both wild type and the opy2 ssk1 mutant carrying the empty vector, the ScOPY2-myc or CaOPY2-myc expression vectors were challenged with $0.9 \mathrm{M} \mathrm{NaCl}$ and samples were taken at different time points. Hog 1 phosphorylation was detected in the wild type strain exposed to osmotic stress and in the opy2 ssk1mutant expressing the ScOpy2-myc protein, nevertheless the opy2 ssk1 mutant was unable to phosphorylate the MAPK Hog1 either carrying the empty vector or the CaOpy2-myc protein (Fig. 4C). All these results suggest that $\mathrm{CaOpy} 2$ is unable to signal an increase of external osmolarity to Hog1 when it is expressed in S. cerevisiae although the overexpression of $\mathrm{CaOpy} 2$ partially complements the osmosensitivity of a S. cerevisiae opy2 ssk1 mutant either on solid or in liquid media. 


\section{CaOpy2 and ScOpy2 display different post-translational modifications}

ScOpy2 is a transmembrane protein that becomes manosylated by the O-mannosyltransferase Pmt4 protein in a Ser-rich (SR) region lying in its extracellular domain (Yang et al., 2009). Although the theoretical molecular weight of ScOpy2-myc is $40.354 \mathrm{kDa}$, this protein migrates at around $100 \mathrm{kDa}$ in a $12 \%$ SDS-PAGE gel (Yang et al., 2009). The predicted molecular weight of CaOpy2-myc is $58.65 \mathrm{kDa}$. However, $\mathrm{CaOpy} 2$ expressed in S. cerevisiae displayed a molecular weight close to $75 \mathrm{kDa}$. This result suggests a lower level of glycosylation on the CaOpy2. The mobility of CaOpy2 did not change when the gene was expressed in a S. cerevisiae wild type or opy2 ssk1or in a C. albicans opy2 mutant (Fig. 5 and data not shown). CaOpy2 may suffer similar post-translational modification in both microorganisms. In addition, no differences in size were detected when CaOPY2-myc was expressed in pmt1, pmt2/PMT2, and pmt4 C. albicans mutants (Fig. 5C). This result correlates with the absence in CaOPY2 of the Ser rich domains described in S. cerevisiae, suggesting that it is not O-manosylated or its manosylation does not depend on the Pmts analysed.

\section{Decreased virulence of Caopy2 mutant using the Galleria mellonella candidiasis model}

The larvae of Galleria mellonella, commonly known as Greater Wax Moth or Honeycomb Moth, can be used as invertebrate host to study virulence. $10^{6}$ Candida cells were injected in PBS at the last left pro-leg directly into the hemocoel and dead larvae were monitored along the time. Figure $6 \mathrm{~A}$ shows the survival curves, the opy2 mutant displayed a decreased virulence compared to the wild type and reintegrant strains. 3 days after inoculation $70 \%$ of the larvae inoculated with the opy2 mutant remain viable while $100 \%$ of the CAF2 inoculated larvae died. Significant differences were evidenced using a Kaplan-Meier statistical analysis. These data 
suggest the involvement of Opy2 in C. albicans virulence. We also used the human promyelocytic cell line HL-60 (Mullick et al., 2004). This cell line was differentiated to neutrophils with DMSO for 4 days. C. albicans cells were then were co-incubated with HL-60 at a multiplicity of infection (MOI) of 40 granulocytes per Candida cell for $2 \mathrm{~h}$. Mammalian cells were broken down and C.albicans viability was determined by CFUs count. No significant differences were observed among the strains analyzed (Fig. 6B). The opy2 mutant displayed a comparable survival percentage to wild type or reintegrant mutant suggesting that CaOpy2 is not required for the survival in the presence of this cellular line.

\section{Discussion}

The MAPK signal transduction pathways are important mechanisms of adaptation to external changes. Although traditionally they are considered as "pathways" they constitute a real network of interconnected proteins. Nevertheless, the specificity of the response to the original stimulus is required. In this sense, the general draft of the MAPK organization increases its complexity when the knowledge about their signaling mechanisms and regulation improves. These signaling routes have evolved to adapt cells to their external environment and, therefore the may differ from organisms adapted to different ecological niches.

In this work the transmembrane protein Opy2 has been analyzed in the fungal pathogen C. albicans. The phenotypical characterization of the defective mutant revealed that this protein plays a crucial role in the signaling to the MAPK Cek1. This MAP kinase belongs to the socalled SVG pathway (Sterile-Vegetative Growth) thus, this pathway is involved in the cell wall biogenesis (Roman et al., 2005). Additionally, Cek1 also belong to a new pathway namely the white-cell pheromone response involved in the biofilm formation and the opaque-cell pheromone response pathways implicated in mating (Soll, 2011). The MAPK module is also involved in quorum sensing and virulence (Csank et al., 1998; Roman et al., 2005; Roman et al., 
2009b; Roman et al., 2009a; Yi et al., 2011). Although the MAPK core remains invariable, upstream elements and effectors differ depending on the stimulus and the response generated. In fact, the white-phase pheromone response and the opaque-cell pheromone response pathways shared upstream elements: Ste2 or Ste3 as sensors, the heterotrimeric G protein formed by Cag1, Ste18 and Ste4 and the kinase Cst20. These pathways differ in the transcription factor and the response generated: Tec1 belong to the white-phase pheromone response and $\mathrm{Cph} 1$ to the opaque-cell pheromone response pathway.

Concerning the role of Cek1 in the biogenesis of the cell wall several works reported the implication of Sho1 and Msb2 as upstream components required to phosphorylate Cek1 in response to resumption of growth in fresh medium, disturbing cell wall agents such as tunycamin and Congo red or mutant defective in manosylation (Cantero et al., 2007; Roman et al., 2005; Roman et al., 2009b; Roman et al., 2009a). In the present work we stated the implication of another transmembrane protein, Opy2, in the signaling to Cek1 (Fig. 7B). The currently reported results suggest that this protein is involved in the biogenesis of the cell wall and in the resumption of growth in fresh medium, since it is under these conditions where the lack of Opy2 completely prevents Cek1 phosphorylation. Surprisingly, opy2 mutants were more sensitive to Congo red but not to tunicamyn or caspofungine on solid media. In this case, the signaling absence does not correlate with sensitivity on plate. The different behavior could point towards to the different mechanisms played by the different stimuli analyzed and, perhaps the role played by others signaling pathways in the response to specific compounds. Thus, in S. cerevisiae the transcriptional response to different cell wall stresses such as Congo red, caspofungin, and zymolyase is specific of the compound and involves different sensors and MAPK cascades mainly cell-wall integrity and HOG pathways (Bermejo et al., 2008; Garcia et al., 2004; Garcia et al., 2009). In C. albicans, two MAP kinases become activates upon cell wall disturbing compounds: Cek1 and Mkc1. In this work we have focused on Cek1 and its 
dependency on Opy2 transmembrane protein. In S. cerevisiae, it has been reported that Slt2 became phosphorylated in the presence of zymolyase and this activation required a functional HOG pathway, among other elements, Opy2 is required for this activation (Bermejo et al., 2008). Nevertheless, no phosphorylation of Kss1 has been reported under these experimental conditions. In our hand, the lack of OPY2 in S. cerevisiae did not prevent Kss1 phosphorylation (data not shown) suggesting evolved differences among MAPK signaling in S. cerevisiae and C. albicans.

As mentioned before, the Opy2 transmembrane protein takes part in the HOG pathway triggering the signal to Hog1 in response to osmotic stress in S. cerevisiae (Yamamoto et al., 2010) (Fig. 7B). In this microorganism, Opy 2 seems to play a relevant role controlling the specificity of the signal transmitted. It has been identified the conserved domain (CR-B) through which Opy2 binds to Ste50. The affinity between Opy2 and Ste50 increased when this CR-B domain is phosphorylated by Yck1/Yck2 in the presence of glucose and then, it is able to trigger the signal to Hog1 upon an external osmolarity increase (Yamamoto et al., 2010). Comparing the OPY2 sequences of both microorganisms, C. albicans and S. cerevisiae, three regions, A, C and D identified in S. cerevisiae are practically identical in the CaOpy2 (Fig. 7A). Nevertheless, the mentioned domain CR-B involved in binding to Ste50 is not present in C. albicans suggesting signaling differences between both microorganisms. When the CaOPY2 gene was over-expressed in S. cerevisiae, it was able to partially complement the osmosensitivity of the opy2 ssk1 double mutant either on solid or liquid media. Nevertheless, no Hog1 phosphorylation was detected under osmotic challenge in the S. cerevisiae opy2 ssk1 double mutant carrying the CaOpy2-myc-pCM189. This data supports the implication of the CR-B in the signaling to Hog1 in S. cerevisiae, and could explain why the absence of such region in $\mathrm{CaOpy} 2$ prevents $\mathrm{Hog} 1$ phosphorylation even when $\mathrm{CaOpy} 2$ is expressed in S. cerevisiae. Moreover, the deletion of OPY2 in C. albicans did not alter the sensitivity to 
osmotic or oxidative stress, neither the Hog1 phosphorylation pattern nor the response generated (STL1 expression and glycerol accumulation). Taken all these data together we can conclude that Opy2 has no role in the HOG pathway in C. albicans (Fig. 7B).

The partial complementation of the osmosensitivity observed in opy2 ssk1 mutant overexpressing CaOpy 2 may be due to the over-expression and thus, a possibly cross-talk or it could be due to the function played by others signaling pathways. Currently, the role played by Cek1mediated pathway in this complementation is under investigation.

Although, the results reported in the present work did not demonstrate that the lack of the CR-B region is the responsible for the lack of Hog1 phosphorylation. We could exclude others reason such as the different post-translational modifications. Although it remains unclear what kind of modifications may suffer the CaOpy2 protein, these seem to be similar when the protein is expressed either in C. albicans or S. cerevisiae since the electrophoretic pattern is comparable in both organisms. CaOpy2 has a putative transmembrane domain and therefore it must be glycosilated. The expression of the tagged myc version of the CaOpy2 in mutants lacking different C. albicans Pmts (protein O-mannosyltransferase) did not alter the electrophoretic distance suggesting that the Pmts analyzed are not required to O-manosylated CaOpy2. ScOPY2 has two predicted glycosilated Ser-rich (SR) regions, SR1 (aa 2-22) and SR2 (aa 75-84) in its extracellular domain, Pmt4 modifies the SR1 region in ScOpy2 (Yang et al., 2009). These putative glycosilation sites are not observable in the C. albicans homologous Opy2 accordingly the post-transcriptional modifications may differ from ScOpy2.

The treatment with tunicamycin, a compound that blocks the synthesis of all N-linked glycoproteins, did not shed light on the modification suffered by CaOpy2 given that no differences in the CaOpy2-myc electrophoretic distance were detected when cultures were treated with this compound (data not shown). 
Another important task to be addressed in C. albicans is its virulence. Several models have been developed to study the virulence of C. albicans, the mammalian models are the most common but at the same time expensive and ethically compromised. For these reasons alternative model are currently increasing its popularity (Brennan et al., 2002; Desalermos et al., 2012; Mylonakis, 2008). The larvae of Galleria mellonella is easy to purchase and inoculate and provide results within $48 \mathrm{~h}$ depending on the inoculated doses (Cotter et al., 2000; Fuchs et al., 2010). This model is a convenient insect model that allows assessing the virulence of microbial pathogens given comparable results to the results obtained through systemic infection of mice (Brennan et al., 2002; Desalermos et al., 2012; Mylonakis, 2008). The opy2 mutant displayed a reduced virulence compare to the wild type and reintegrant strains in the G. mellonella model as it was evidenced using a Kaplan-Meier statistic analysis. Others mutants belonging to the SVG pathways (cpp1, cek1, hst7 and cst20) have been investigated using this insect model and comparing with previous studies in mice (Brennan et al., 2002). In general the results displayed a good correlation between both models although the differences among mutants and wild type were less evident in the G. mellonella model. This study concluded that Galleria may provide a convenient and inexpensive model for the in vivo screening of mutants of C. albicans. Following this advice we have replaced the use of mice for G. mellonella to evidence that Opy2 plays a role in virulence.

Primary cultures and mammalian cell line are also useful models to analyze susceptibility to phagocytic cells. The HL-60 is a promyelocytic cell line proved to be useful for the analysis of the granulocyte responses against C. albicans (Mullick et al., 2004). Using this model, the cek1 mutant displayed a killing similar to the wild type (Arana et al., 2007), in spite of being less virulent in the murine systemic infection and G. mellonella models . In this work, we have displayed similar result for the opy2 mutant. This mutant is less virulent in the insect G. mellonella model but displayed wild type-like susceptibility to granulocytes HL-60. These 
results support once more that Opy2 belongs to the same pathway than Hst7 and Cek1 and evidence differences among the experimental model assayed.

Our data clearly evidenced the role of CaOpy 2 in Cek1 phosphorylation and, therefore, cell wall remodeling. We have also demonstrated that $\mathrm{CaOpy} 2$ does not signal to Hog1 upon osmotic stress in C. albicans. These two facts differ significantly from the role played by Opy2 in S. cerevisiae and suggest evolutionary differences among signal transduction mechanisms between these two microorganisms, C. albicans and S. cerevisiae (Fig.7B).

\section{Acknowledgements}

We thank to T.J.C. Caldas for her contribution to the initial stage of the present work. We thank Dr. J. Ernst and Dr. C. Wu for generously providing Pmts mutant and S. cerevisiaes trains, respectively. The caspofungine was kindly provided by MSD. This work is supported by GrantsBio2006-03637, BIO2009-07788 and PIM2010EPA-00658 (from Ministerio de Educación y Ciencia). 


\section{References}

Alonso-Monge,R., Carvaihlo,S., Nombela,C., Rial,E., and Pla,J. (2009). The Hog1 MAP kinase controls respiratory metabolism in the fungal pathogen Candida albicans. Microbiol. 155, 413423.

Alonso-Monge,R., Navarro-García,F., Molero,G., Diez-Orejas,R., Gustin,M., Pla,J., Sánchez,M., and Nombela,C. (1999). Role of the mitogen-activated protein kinase Hog1p in morphogenesis and virulence of Candida albicans. J. Bacteriol. 181, 3058-3068.

Alonso-Monge,R., Navarro-García,F., Román,E., Negredo,A.I., Eisman,B., Nombela,C., and Pla,J. (2003). The Hog1 Mitogen-Activated Protein Kinase Is Essential in the Oxidative Stress Response and Chlamydospore Formation in Candida albicans. Eukaryot. Cell 2, 351-361.

Alonso-Monge,R., Real,E., Wojda,I., Bebelman,J.P., Mager,W.H., and Siderius,M. (2001). Hyperosmotic stress response and regulation of cell wall integrity in Saccharomyces cerevisiae share common functional aspects. Mol. Microbiol. 41, 717-730.

Arana,D.M., Alonso-Monge,R., Du,C., Calderone,R., and Pla,J. (2007). Differential susceptibility of mitogen-activated protein kinase pathway mutants to oxidative-mediated killing by phagocytes in the fungal pathogen Candida albicans. Cell Microbiol.

Arana,D.M., Nombela,C., Alonso-Monge,R., and Pla,J. (2005). The Pbs2 MAP kinase kinase is essential for the oxidative-stress response in the fungal pathogen Candida albicans. Microbiol. $151,1033-1049$.

Bermejo,C., Rodriguez,E., Garcia,R., Rodriguez-Pena,J.M., de la Concepcion,M.L.R., Rivas,C., Arias,P., Nombela,C., Posas,F., and Arroyo,J. (2008). The sequential activation of the yeast 
HOG and SLT2 pathways is required for cell survival to cell wall stress. Mol. Biol. Cell 19, $1113-1124$.

Brennan,M., Thomas,D., Whiteway,M., and Kavanagh,K. (2002). Correlation between virulence of Candida albicans mutants in mice and Galleria mellonella larvae. FEMS Immunol. Med. Microbiol. 34, 153.

Calera,J.A., Zhao,X.J., and Calderone,R. (2000). Defective hyphal development and avirulence caused by a deletion of the SSK1 response regulator gene in Candida albicans. Infect. Immun. $68,518-525$

Cantero,P.D., Lengsfeld,C., Prill,S.K., Subanovic,M., Roman,E., Pla,J., and Ernst,J.F. (2007). Transcriptional and physiological adaptation to defective protein-O-mannosylation in Candida albicans. Mol. Microbiol. 64, 1115-1128.

Chauhan,N., Inglis,D., Roman,E., Pla,J., Li,D., Calera,J.A., and Calderone,R. (2003). Candida albicans response regulator gene SSK1 regulates a subset of genes whose functions are associated with cell wall biosynthesis and adaptation to oxidative stress. Eukaryot. Cell 2, 10181024.

Cheetham,J., Smith,D.A., da Silva,D.A., Doris,K.S., Patterson,M.J., Bruce,C.R., and Quinn,J. (2007). A Single MAPKKK Regulates the Hog1 MAPK Pathway in the Pathogenic Fungus Candida albicans. Mol. Biol. Cell 18, 4603-4614.

Chen,D.C., Yang,B.C., and Kuo,T.T. (1992). One-step transformation of yeast in stationary phase. Curr. Genet. 21, 83-84.

Chen,J., Chen,J., Lane,S., and Liu,H. (2002). A conserved mitogen-activated protein kinase pathway is required for mating in Candida albicans. Mol. Microbiol. 46, 1335-1344. 
Cotter,G., Doyle,S., and Kavanagh,K. (2000). Development of an insect model for the in vivo pathogenicity testing of yeasts. Fems Immunology and Medical Microbiology 27, 163-169.

Csank,C., Schröppel,K., Leberer,E., Harcus,D., Mohamed,O., Meloche,S., Thomas,D.Y., and Whiteway,M. (1998). Roles of the Candida albicans mitogen-activated protein kinase homolog, Cek1p, in hyphal development and systemic candidiasis. Infect. Immun. 66, 2713-2721.

Desalermos,A., Fuchs,B.B., and Mylonakis,E. (2012). Selecting an Invertebrate Model Host for the Study of Fungal Pathogenesis. Plos Pathogens 8.

Diez-Orejas,R., Molero,G., Navarro-García,F., Pla,J., Nombela,C., and Sánchez-Pérez,M. (1997). Reduced virulence of Candida albicans MKC1 mutants: a role for a mitogen-activated protein kinase in pathogenesis. Infect. Immun. 65, 833-837.

Eisman,B., Alonso-Monge,R., Román,E., Arana,D., Nombela,C., and Pla,J. (2006). The Cek1 and Hog1 Mitogen-Activated Protein Kinases Play Complementary Roles in Cell Wall Biogenesis and Chlamydospore Formation in the Fungal Pathogen Candida albicans. Eukaryot. Cell. 5, 347-358.

Ekiel,I., Sulea,T., Jansen,G., Kowalik,M., Minailiuc,O., Cheng,J., Harcus,D., Cygler,M., Whiteway,M., and Wu,C. (2009). Binding the atypical RA domain of Ste50p to the unfolded Opy2p cytoplasmic tail is essential for the high-osmolarity glycerol pathway. Mol. Biol. Cell $20,5117-5126$

Enjalbert,B., Smith,D.A., Cornell,M.J., Alam,I., Nicholls,S., Brown,A.J., and Quinn,J. (2006). Role of the Hog1 stress-activated protein kinase in the global transcriptional response to stress in the fungal pathogen Candida albicans. Mol. Biol. Cell. 17, 1018-1032. 
Fonzi,W.A. and Irwin,M.Y. (1993). Isogenic strain construction and gene mapping in Candida albicans. Genetics 134, 717-728.

Fuchs,B.B., O'Brien,E., El Khoury,J.B., and Mylonakis,E. (2010). Methods for using Galleria mellonella as a model host to study fungal pathogenesis. Virulence 1, 475-482.

Garcia,R., Bermejo,C., Grau,C., Perez,R., Rodriguez-Pena,J.M., Francois,J., Nombela,C., and Arroyo,J. (2004). The global transcriptional response to transient cell wall damage in Saccharomyces cerevisiae and its regulation by the cell integrity signaling pathway. J. Biol. Chem. 279, 15183-15195.

Garcia,R., Rodriguez-Pena,J.M., Bermejo,C., Nombela,C., and Arroyo,J. (2009). The High Osmotic Response and Cell Wall Integrity Pathways Cooperate to Regulate Transcriptional Responses to Zymolyase-induced Cell Wall Stress in Saccharomyces cerevisiae. J. Biol. Chem. 284, 10901-10911.

Gari,E., Piedrafita,L., Aldea,M., and Herrero,E. (1997). A set of vectors with a tetracyclineregulatable promoter system for modulated gene expression in Saccharomyces cerevisiae. Yeast $13,837-848$

Kultz,D. (1998). Phylogenetic and functional classification of mitogen- and stress-activated protein kinases. J. Mol. Evol. 46, 571-588.

Kumamoto,C.A. (2005). A contact-activated kinase signals Candida albicans invasive growth and biofilm development. Proc. Natl. Acad. Sci. U. S. A 102, 5576-5581.

Lee,B.N. and Elion,E.A. (1999). The MAPKKK Ste11 regulates vegetative growth through a kinase cascade of shared signaling components. Proc Natl Acad Sci U. S. A 96, 12679-12684. 
Martin,H., Rodriguez-Pachon,J.M., Ruiz,C., Nombela,C., and Molina,M. (2000). Regulatory mechanisms for modulation of signaling through the cell integrity Slt2-mediated pathway in Saccharomyces cerevisiae. J. Biol. Chem. 275, 1511-1519.

Mullick,A., Elias,M., Harakidas,P., Marcil,A., Whiteway,M., Ge,B., Hudson,T.J., Caron,A.W., Bourget,L., Picard,S., Jovcevski,O., Massie,B., and Thomas,D.Y. (2004). Gene expression in HL60 granulocytoids and human polymorphonuclear leukocytes exposed to Candida albicans. Infect. Immun. 72, 414-429.

Mylonakis,E. (2008). Galleria mellonella and the study of fungal pathogenesis: Making the case for another genetically tractable model host. Mycopathologia 165, 1-3.

Navarro-García,F., Alonso-Monge,R., Rico,H., Pla,J., Sentandreu,R., and Nombela,C. (1998). A role for the MAP kinase gene $M K C 1$ in cell wall construction and morphological transitions in Candida albicans. Microbiol. 144, 411-424.

Navarro-Garcia,F., Eisman,B., Fiuza,S.M., Nombela,C., and Pla,J. (2005). The MAP kinase Mkc1p is activated under different stress conditions in Candida albicans. Microbiol. 151, 27372749.

Navarro-García,F., Sanchez,M., Pla,J., and Nombela,C. (1995). Functional characterization of the MKC1 gene of Candida albicans, which encodes a mitogen-activated protein kinase homolog related to cell integrity. Mol. Cell. Biol. 15, 2197-2206.

O'Rourke,S.M. and Herskowitz,I. (2002). A third osmosensing branch in Saccharomyces cerevisiae requires the Msb2 protein and functions in parallel with the Sho1 branch. Mol. Cell Biol. 22, 4739-4749.

Odds,F.C. (1988). Candida and candidosis. (London: Baillière Tindall). 
Park,Y.N. and Morschhauser,J. (2005). Tetracycline-inducible gene expression and gene deletion in Candida albicans. Eukaryot. Cell 4, 1328-1342.

Posas,F., Wurgler-Murphy,S.M., Maeda,T., Witten,E.A., Thai,T.C., and Saito,H. (1996). Yeast HOG1 MAP kinase cascade is regulated by a multistep phosphorelay mechanism in the SLN1YPD1-SSK1 "two-component" osmosensor. Cell 86, 865-875.

Reuss,O., Vik,A., Kolter,R., and Morschhauser,J. (2004). The SAT1 flipper, an optimized tool for gene disruption in Candida albicans. Gene. 341:119-27., 119-127.

Roman,E., Alonso-Monge,R., Gong,Q.H., Li,D.M., Calderone,R., and Pla,J. (2009a). The Cek1 MAPK is a short-lived protein regulated by quorum sensing in the fungal pathogen Candida albicans. Fems Yeast Research 9, 942-955.

Roman,E., Cottier,F., Ernst,J.F., and Pla,J. (2009b). Msb2 signaling mucin controls activation of Cek1 mitogen-activated protein kinase in Candida albicans. Eukaryot. Cell 8, 1235-1249.

Román,E., Nombela,C., and Pla,J. (2005). The Sho1 adaptor protein links oxidative stress to morphogenesis and cell wall biosynthesis in the fungal pathogen Candida albicans. Mol. Cell Biol. 25, 10611-10627.

San José,C., Alonso-Monge,R., Pérez-Díaz,R.M., Pla,J., and Nombela,C. (1996). The mitogenactivated protein kinase homolog HOG1 gene controls glycerol accumulation in the pathogenic fungus Candida albicans. J. Bacteriol. 178, 5850-5852.

Smith,D.A., Nicholls,S., Morgan,B.A., Brown,A.J., and Quinn,J. (2004). A Conserved StressActivated Protein Kinase Regulates a Core Stress Response in the Human Pathogen Candida albicans. Mol. Biol. Cell .. 
Soll,D.R. (2011). Evolution of a new signal transduction pathway in Candida albicans. Trends Microbiol. 19, 8-13.

Tatebayashi,K., Tanaka,K., Yang,H.Y., Yamamoto,K., Matsushita,Y., Tomida,T., Imai,M., and Saito,H. (2007). Transmembrane mucins Hkr1 and Msb2 are putative osmosensors in the SHO1 branch of yeast HOG pathway. EMBO J. 26, 3521-3533.

Wu,C.L., Jansen,G., Zhang,L.C., Thomas,D.Y., and Whiteway,M. (2006). Adaptor protein Ste50p links the Ste11p MEKK to the HOG pathway through plasma membrane association. Genes \& Development 20, 734-746.

Yamamoto,K., Tatebayashi,K., Tanaka,K., and Saito,H. (2010). Dynamic control of yeast MAP kinase network by induced association and dissociation between the Ste50 scaffold and the Opy2 membrane anchor. Mol Cell 40, 87-98.

Yang,H.Y., Tatebayashi,K., Yamamoto,K., and Saito,H. (2009). Glycosylation defects activate filamentous growth Kss1 MAPK and inhibit osmoregulatory Hog1 MAPK. EMBO J. 28, 13801391.

Yi,S., Sahni,N., Daniels,K.J., Lu,K.L., Srikantha,T., Huang,G.H., Garnaas,A.M., and Soll,D.R. (2011). Alternative Mating Type Configurations (a/alpha versus a/a or a/alpha) of Candida albicans Result in Alternative Biofilms Regulated by Different Pathways. Plos Biology 9. 


\section{Legend to figures}

Figure 1:Opy2 is involved in cell wall biogenesis. A) Serial dilutions of the indicated C. albicans strains were spotted onto YPD plates (as a control) and YPD supplemented with Congo red to 125 and $250 \mu \mathrm{g} / \mathrm{ml}$. Plates were incubated at 37 and $42^{\circ} \mathrm{C}$ for $24 \mathrm{~h}$ before scanned. B) Plates supplemented with $250 \mu \mathrm{g} / \mathrm{ml}$ Congo red were incubated at $37^{\circ} \mathrm{C}$ for $24 \mathrm{~h}$.

Figure 2: Opy2 is required for Cek1 phosphorylation.A) Exponentially growing cells from the indicated strains (O.D. 1) were exposed to $20 \mu \mathrm{g} / \mathrm{ml}$ Congo red (CR), $2 \mathrm{U} / \mathrm{ml} 100-\mathrm{T}$ zymoliase or $2.5 \mu \mathrm{g} / \mathrm{ml}$ tunicamycin for $1 \mathrm{~h}$. Cells were collected and processed for westernblot. The phosphorylated version of Cek1 was detected using anti-phospho-p44/42antibody (PCek1). Samples from non treated cells are shown as (-). B) Stationary phase cultures (st) from indicated strains were refreshed to 0.2 O.D. in YPD and incubated at $37^{\circ} \mathrm{C}$. Samples were taken 30 minutes after dilution and processed for imnuno-blot analyses. The anti-phosphop44/42antibody was used to detect Cek1 and Mkc1 phosphorylation. C) Stationary growing cells were refreshed into YPD warmed liquid medium and samples were taken after 30 minutes and $1 \mathrm{~h}$ of incubation at $37^{\circ} \mathrm{C}$. Cek1 phosphorylation was detected using the antiphosphop44/42antibody and the anti-actin antibody was used to ensure equally amount of loaded protein. Ten folds cell dilutions of the indicated strains were spotted on YPD plates supplemented or not with $250 \mu \mathrm{g} / \mathrm{ml}$ Congo red (CR) and incubated at $37^{\circ} \mathrm{C}$ for $24 \mathrm{~h}$.

Figure 3: Role of Opy2 in osmotic and oxidative stress signaling. A) Ten fold cell dilutions from the strains indicated were spotted on YPD plates supplemented with $1 \mathrm{M} \mathrm{NaCl}$ or $1.5 \mathrm{M}$ sorbitol. Plates were incubated for $24 \mathrm{~h}$ at $37^{\circ} \mathrm{C}$. B) Exponentially growing cells were challenged with $1 \mathrm{M} \mathrm{NaCl}$ taken samples at different time points (upper panel) or challenge with $1.25 \mathrm{M} \mathrm{NaCl}$ for 10 min (lower panel).(C) Exponentially growing cells were challenged with $10 \mathrm{mM} \mathrm{H}_{2} \mathrm{O}_{2}$. Samples were taken at different time points. Hog1 phosphorylation was 
detected using an Anti-phospho p38 antibody (P-Hog1), anti -actin, anti-Hog1 or anti-Cek1 antibodies were used as control loading.

Figure 4: Role of the CaOpy2 expressed in a S.cerevisiaeopy2 ssk1mutant. A) The BY4741 wild type strain ant its isogenic opy2 ssk1 mutant carriyng the pCM189 empty vector, CaOpy2myc-pCM189 or ScOPY2-myc-pCM189 expression vectors were spotted on YPD plates with $0.5 \mathrm{M} \mathrm{NaCl}$ or $1 \mathrm{M}$ Sorbitol and incubated at $30^{\circ} \mathrm{C}$. Pictures were taken after $24 \mathrm{~h}$ of incubation. B) The indicated strains were growth overnight in YPD or $0.5 \mathrm{M}$ YPD at $30^{\circ} \mathrm{C}$ and pictures were taken under optical microscopy. C)The indicated S. cerevisiae strains were challenged to $0.9 \mathrm{M} \mathrm{NaCl}$ and samples were taken after 0,5 and 15 minutes of incubation at $30^{\circ} \mathrm{C}$ and processed for immuno-blot analyses. Hog 1 phosphorylation was detected using an anti-phospho p38 antibody and anti-Hog1 antibody was used as loading control.

Figure 5: Post-translational modification of ScOpy2 and CaOpy2 proteins. A)The S. cerevisiae wild type strain BY4741carriyng the empty vector (pCM189) or expressing the paralog CaOpy2-myc or the own ScOpy2-myc were disrrupted and processed for Western-blot. Two clones from each transformation were analysed. B) The opy2 C. albicans mutant or S. cerevisiae wild type or opy2 ssk1 mutant carrying the CaOPY2-Myc-pCM189 plasmid were processed for Western-blot analyses. The molecular weight of protein standar is indicated. C) C. albicans opy2 and pmts mutants grown till stationary phase in YPD supplemented with doxicicline to ensure OPY2-myc expression were processed for Western-blot analyses. The CaOpy2-myc and ScOpy2-myc proteins were detected using the anti-Myc 4A6 clone and antiHog1 was used as loading control.

Figure 6: CaOpy2 is involved in pathogenesis. A)Survival curve of G. mellonella larvae injected with $10^{6}$ Candida cells of indicated strains. B) Percentage of viability of C. albicans cells exposed to neutrophil-like cell line HL-60. 1 Candida cell was co-incubated with 40 
neutrophil-like cells for $2 \mathrm{~h}$ at $37^{\circ} \mathrm{C}$ and $5 \% \mathrm{CO}_{2}$. The percentage of viability is shown. Data represent the averages for three independent experiments and the standard deviation (SD).

Figure 7: Comparison between C. albicans and S. cerevisiae. A) Schematic draft of CaOpy2 and ScOpy2, the identified motives or domains are marked; TR means Transmembrane domain, CR, means Conserved Domain and SR, Serine-rich region. B) Diagram of MAPK pathways analyzed in this work. Left panel belong to C. albicans where two independent pathways sense osmotic stress (HOG pathway) and cell wall disturbing compound or resumption of growth (Cek1-mediated pathway). CaOpy2 has been placed in the Cek1-mediated route being involved in cell wall remodeling. The elements that have not been demonstrated experimentally their belonging to the pathway are blur. In S. cerevisiae (right panel), two branches sense osmotic stress and therefore, trigger Hog1 phosphorylation.

\section{Supplementary data}

Figure S1: Susceptibility to tunicamycin and caspofungin. Ten folds cells suspensions from the strains indicated were spotted on YPD plates supplemented with $1 \mu \mathrm{g} / \mathrm{ml}$ and $2 \mu \mathrm{g} / \mathrm{ml}$ tunicamycin (Tn) or $30 \mathrm{ng} / \mathrm{ml}$ and $50 \mathrm{ng} / \mathrm{ml}$ caspofungine $(\mathrm{Cp})$. Plates were incubated for $24 \mathrm{~h}$ at $37^{\circ} \mathrm{C}$.

Figure S2: Susceptibility to oxidative stress different C. albicans strains. 


\section{Table 1:}

Strains used in this work

\begin{tabular}{|c|c|c|c|c|}
\hline \multirow{3}{*}{ Microorganism } & \multirow{3}{*}{ Strain } & \multirow{3}{*}{ Genotype } & \multicolumn{2}{|l|}{ Nomenclature in } \\
\hline & & & Manuscript and & Source \\
\hline & & & Figures & \\
\hline \multirow[t]{2}{*}{ C. albicans } & CAF2-1 & ura3::imm434/ura3::imm434-URA3 & CAF2 (wt) & (Fonzi and Irwin, \\
\hline & & & & 1993) \\
\hline \multirow[t]{2}{*}{ C. albicans } & RM100 & ura3::imm434/ura3::imm434 & RM100 (wt) & (Negredo et al., \\
\hline & & his1::hisG/his1::hisG-URA3-hisG & & 1997) \\
\hline \multirow[t]{2}{*}{ C. albicans } & CHO4-1 & ura3::imm434/ura3::imm434-URA3 & opy 2 & This work \\
\hline & & opy2::FRT/opy2::FRT & & \\
\hline \multirow[t]{3}{*}{ C. albicans } & CHO39 & ura3::imm434/ura3::imm434-URA3 & OPY2 ${ }^{\text {reint }}$ & This work \\
\hline & & opy2::FRT/opy2::FRT ARD1/ard1:: & & \\
\hline & & pOPY2::pOPY2-OPY2-URA3 & & \\
\hline \multirow[t]{4}{*}{ C. albicans } & CHOM-16 & ura3::imm434/ura3::imm434-URA3 & CaOPY2-myc & This work \\
\hline & & opy $2::$ FRT/opy $2:: F R T$ & & \\
\hline & & ADH1/adh1:: tTA pTET-OPY2- & & \\
\hline & & myc-SAT1 & & \\
\hline \multirow[t]{4}{*}{ C. albicans } & ssk1 msb2 sho1 & ura3::imm434/ura3::imm434-URA3 & ssk1 sho1 msb2 & (Román, \\
\hline & & ssk1::hisG/ssk1:: hisG msb2::FRT/ & & 2009b) \\
\hline & & msb2:: FRT sho1::hisG/sho1::hisG- & & \\
\hline & & URA3-hisG & & \\
\hline
\end{tabular}




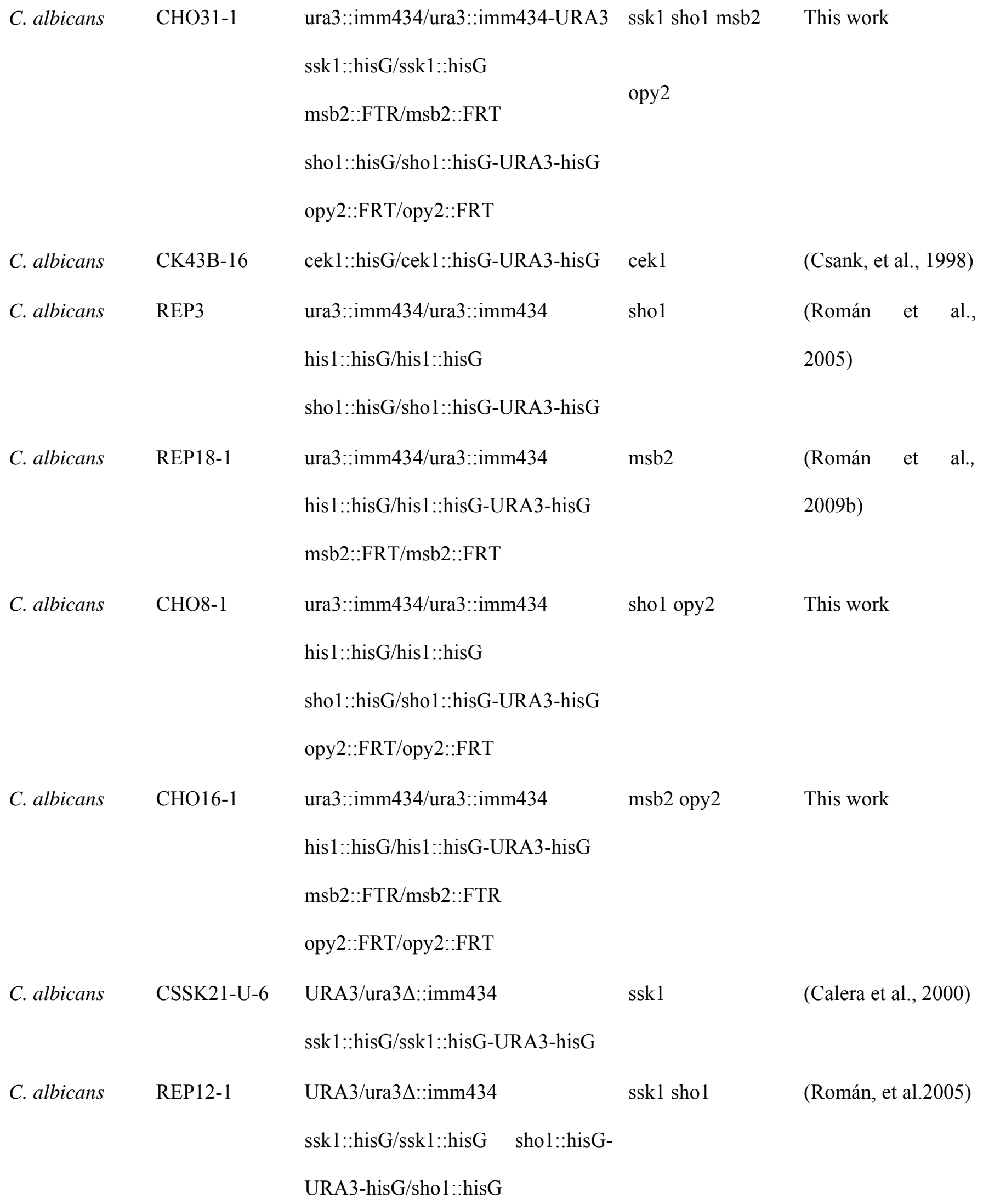




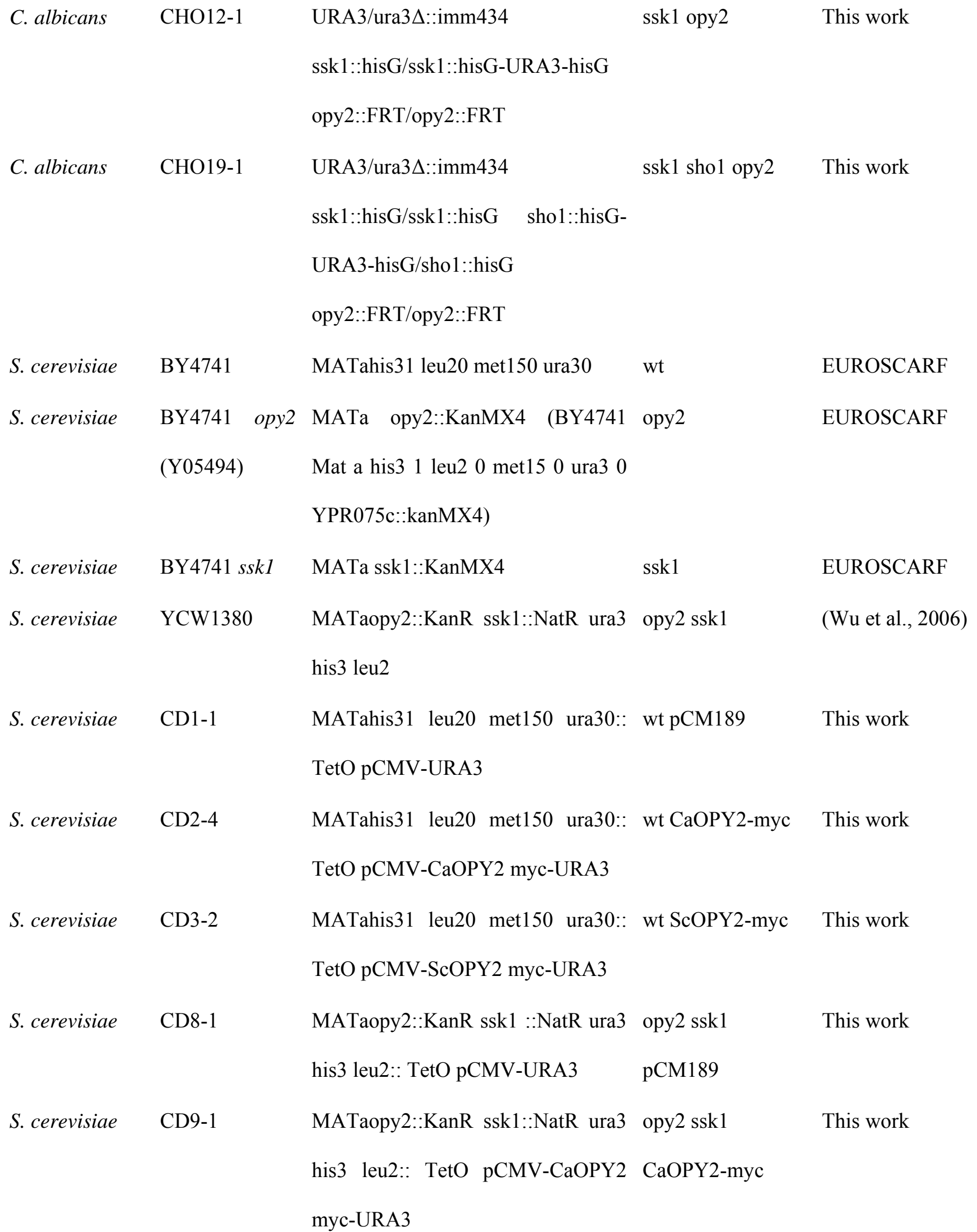


S. cerevisiae CD10-23 MATaopy2::KanR ssk1::NatR ura3 opy2 ssk1

This work

his3 leu2:: TetO pCMV-ScOPY2 ScOPY2-myc

myc-URA3 
A

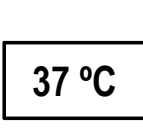

CaOPY2-myc

$42{ }^{\circ} \mathrm{C}$

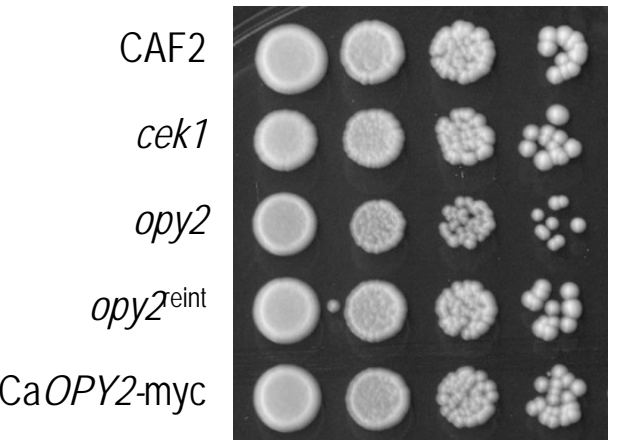

control

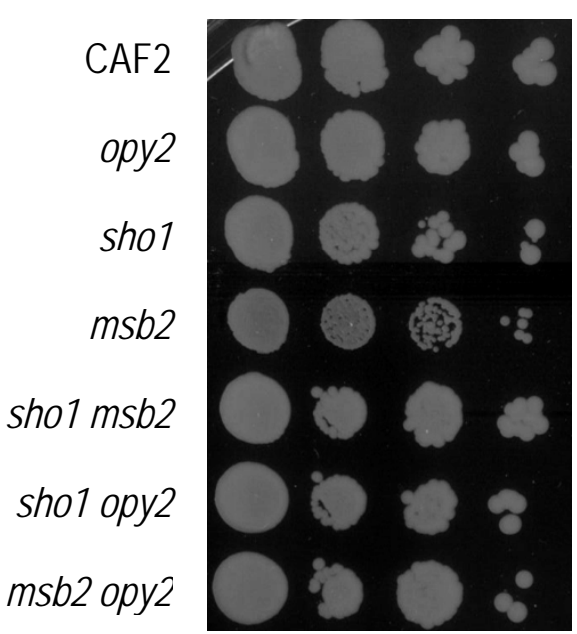

B
$125 \mu \mathrm{g} / \mathrm{ml}$ CR
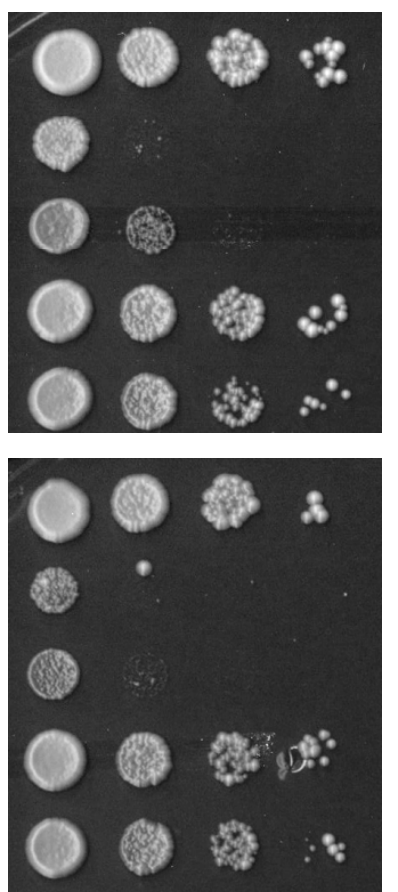

$250 \mu \mathrm{g} / \mathrm{ml} \mathrm{CR}$

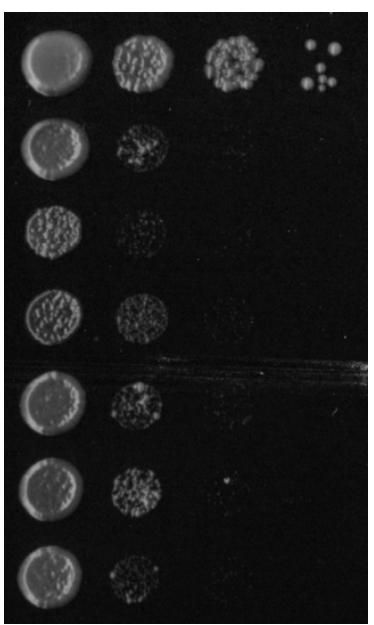

$250 \mu \mathrm{g} / \mathrm{ml} \mathrm{CR}$
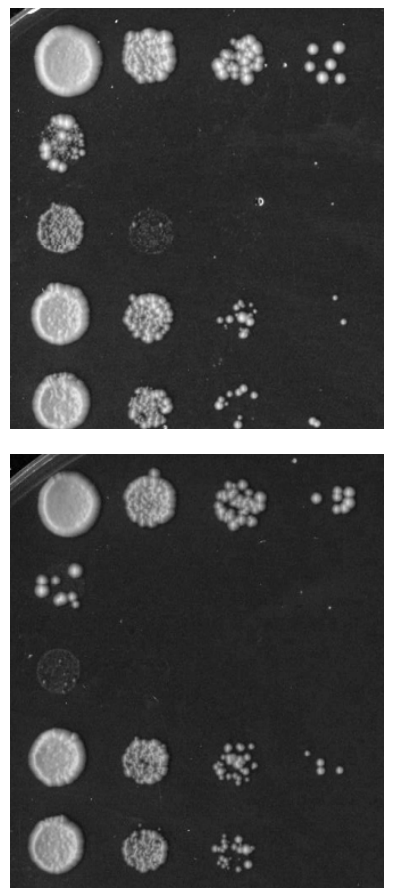

Figure 1 
A

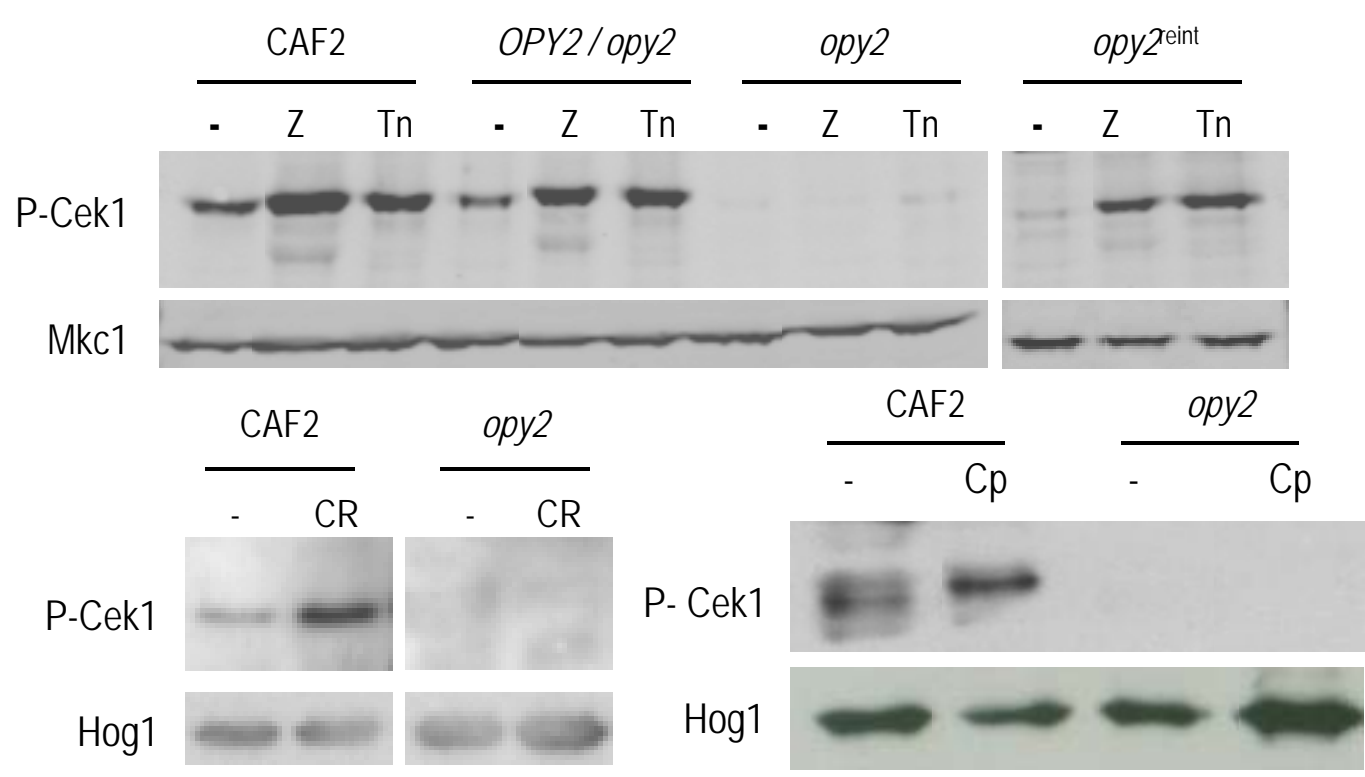

B

$$
\frac{\mathrm{CAF2}}{\mathrm{St} \quad 30^{\prime}} \quad \frac{\mathrm{OPY} 2 / \text { opy2 }}{\mathrm{St} \quad 30^{\prime}} \quad \frac{\frac{0 p y 2}{\mathrm{St}} \quad 30^{\prime}}{\mathrm{St} \quad 30^{\prime}}
$$

P-Mkc1

P-Cek1

Actin

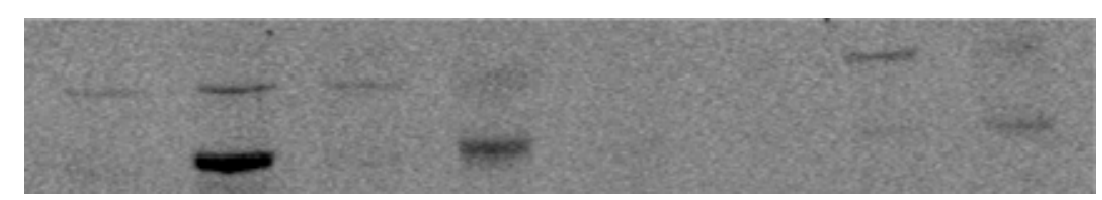

C

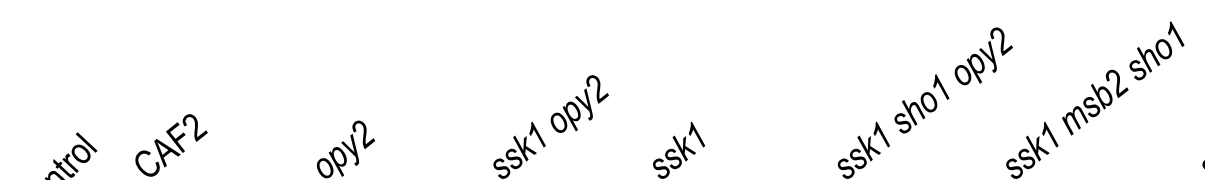

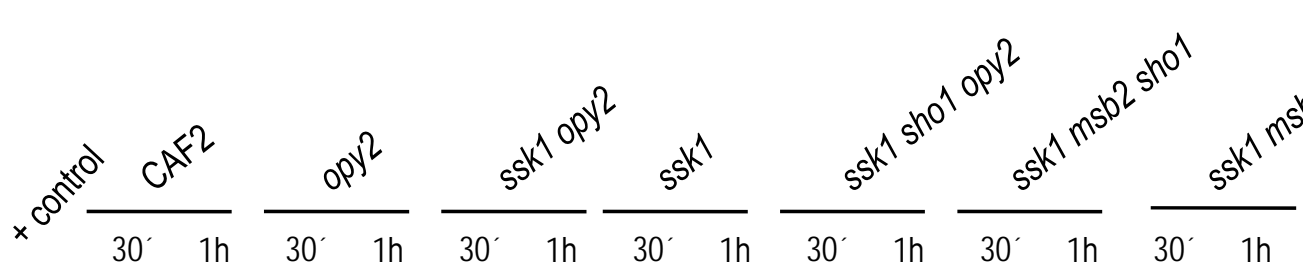

P- Cek1

Actin
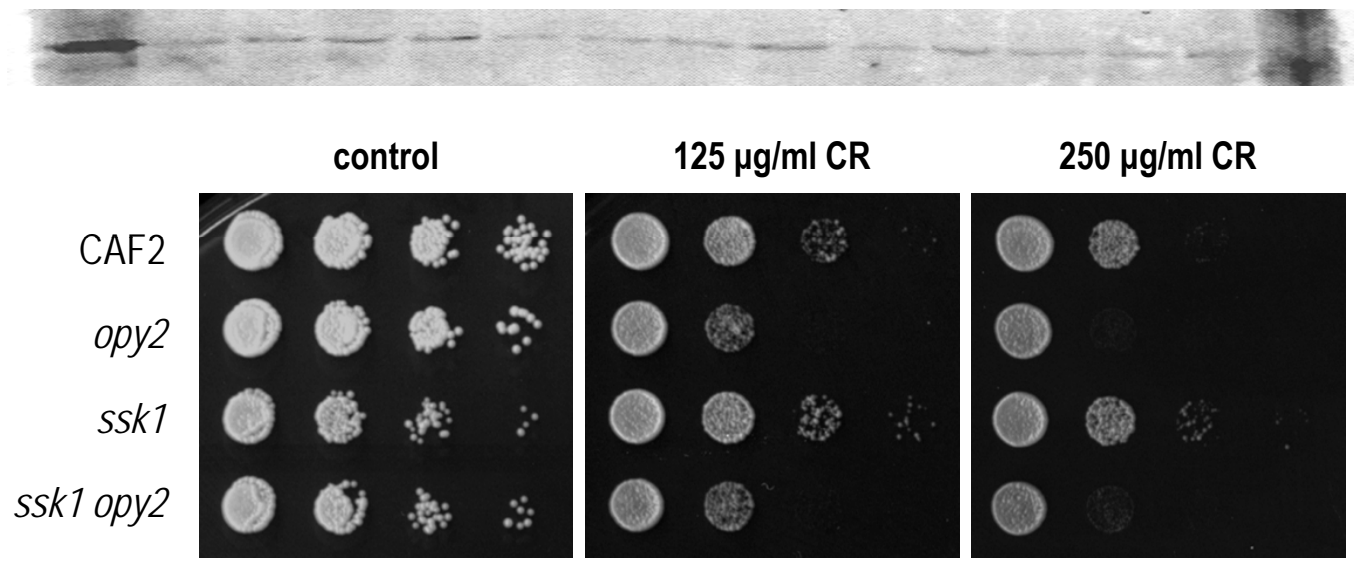

Figure 2 
A

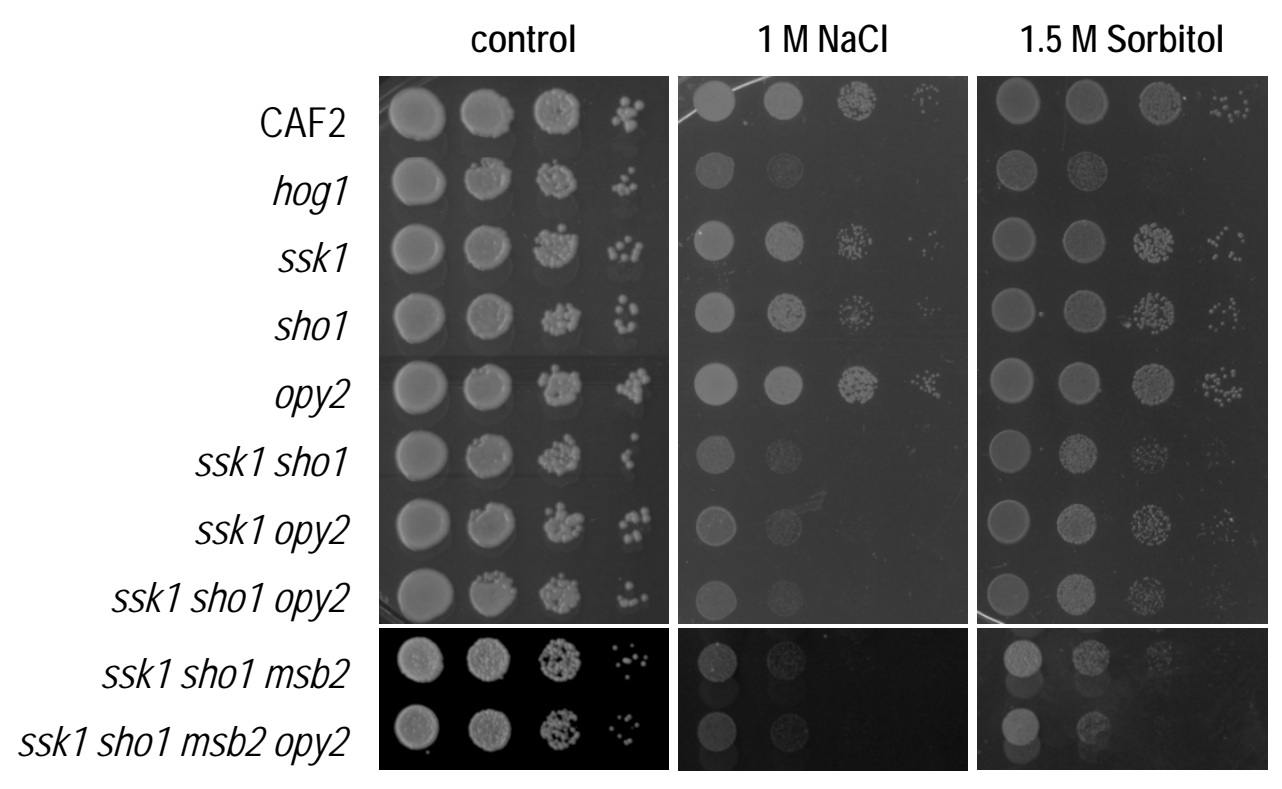

B
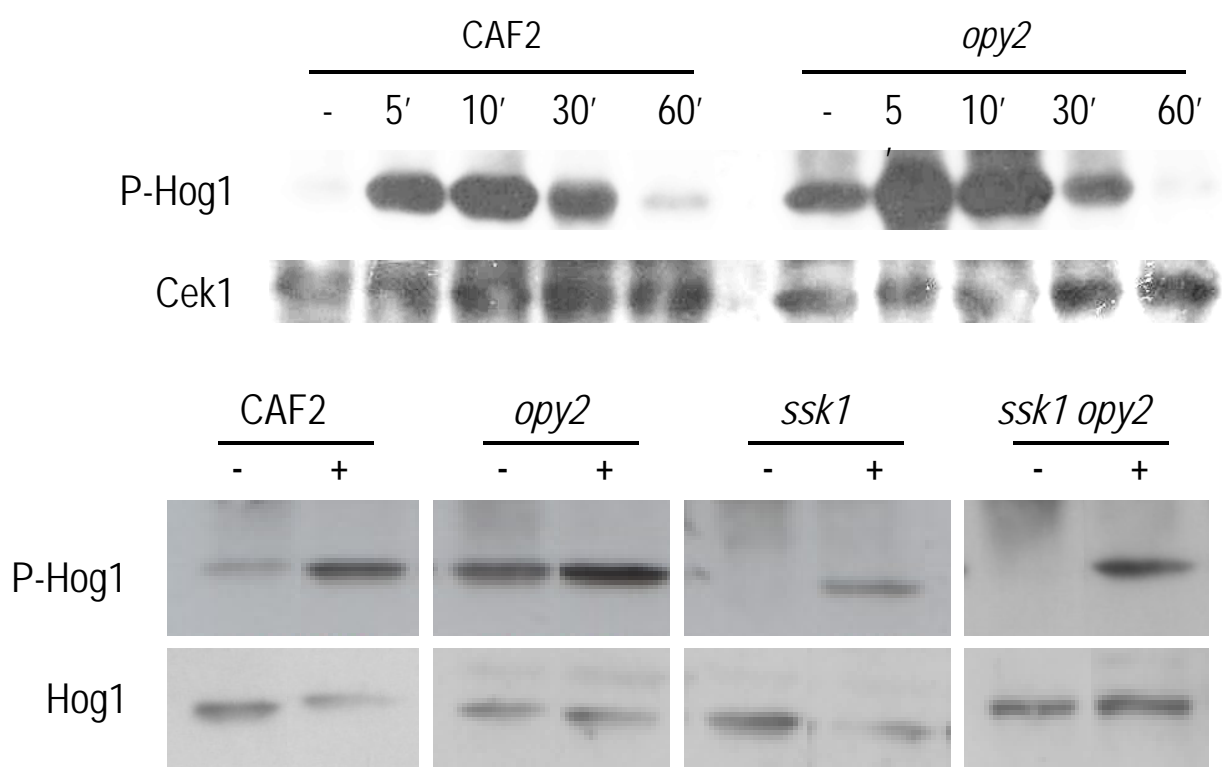

C

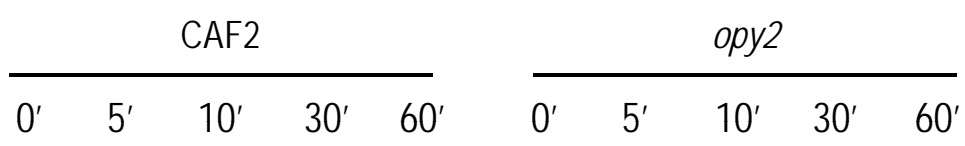

P-Hog1

Actin

Figure 3 
A

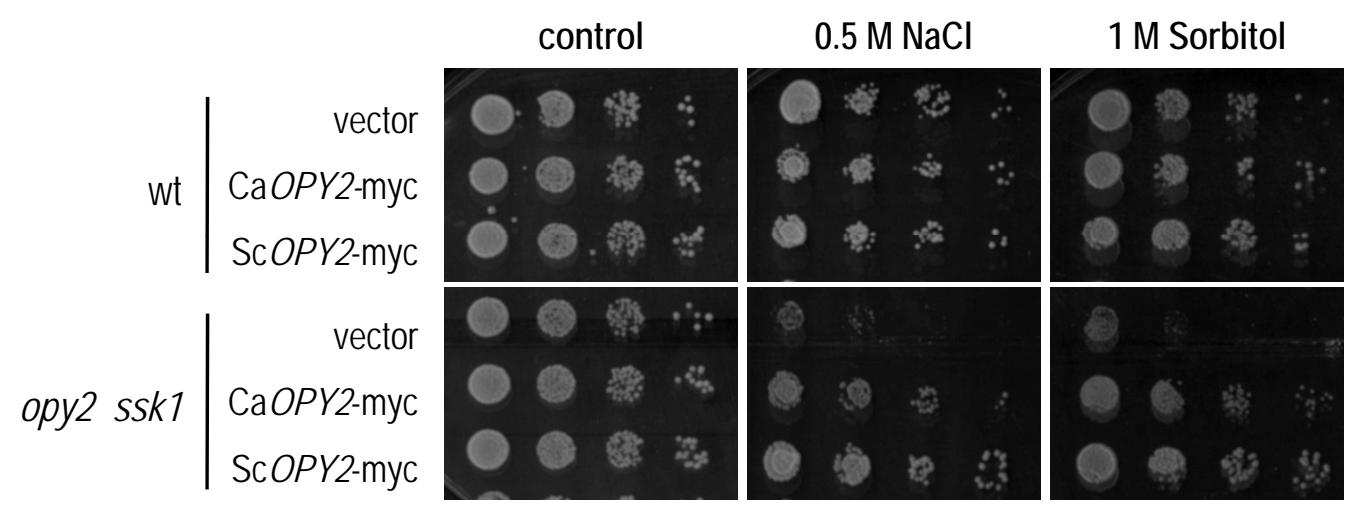

B

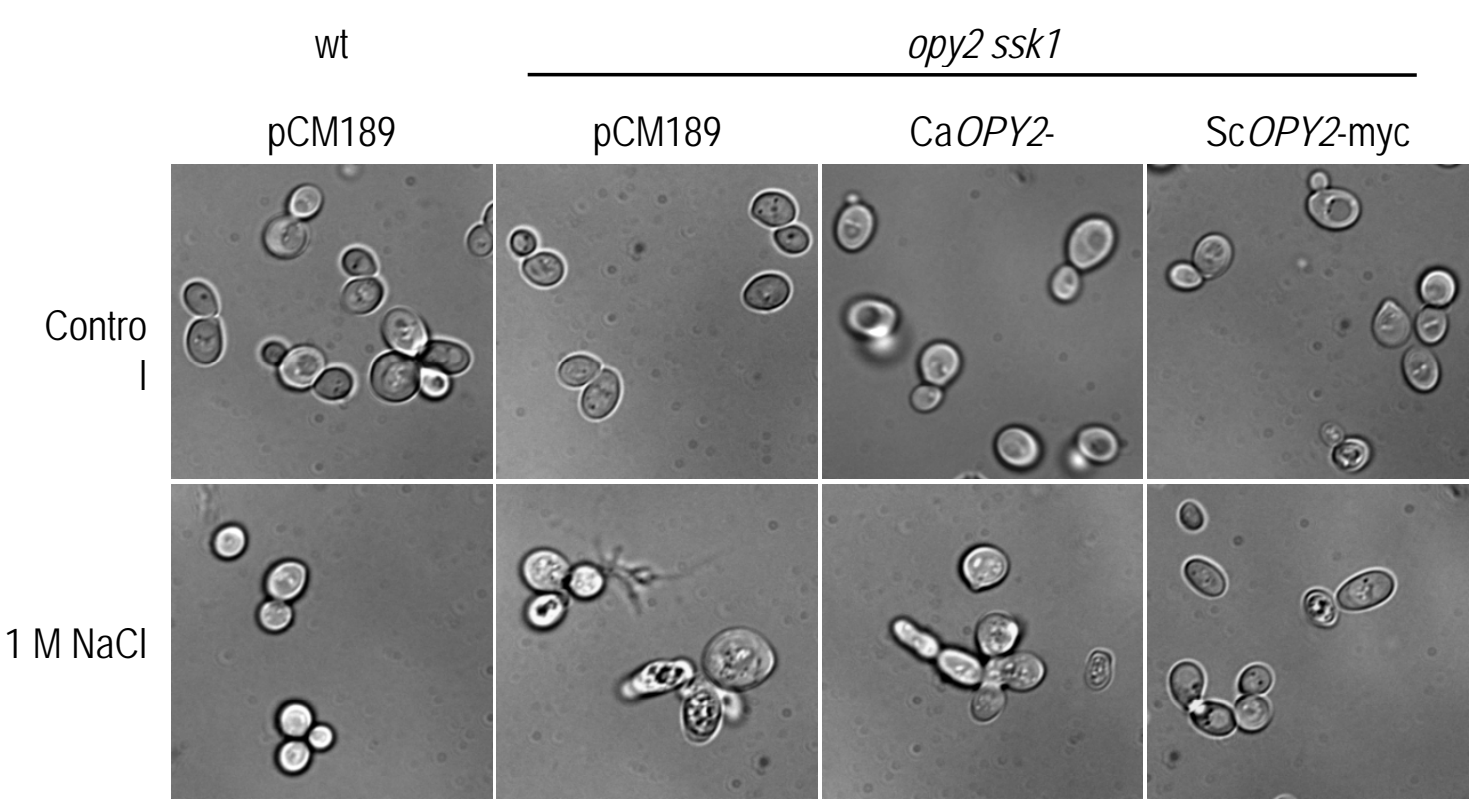

C

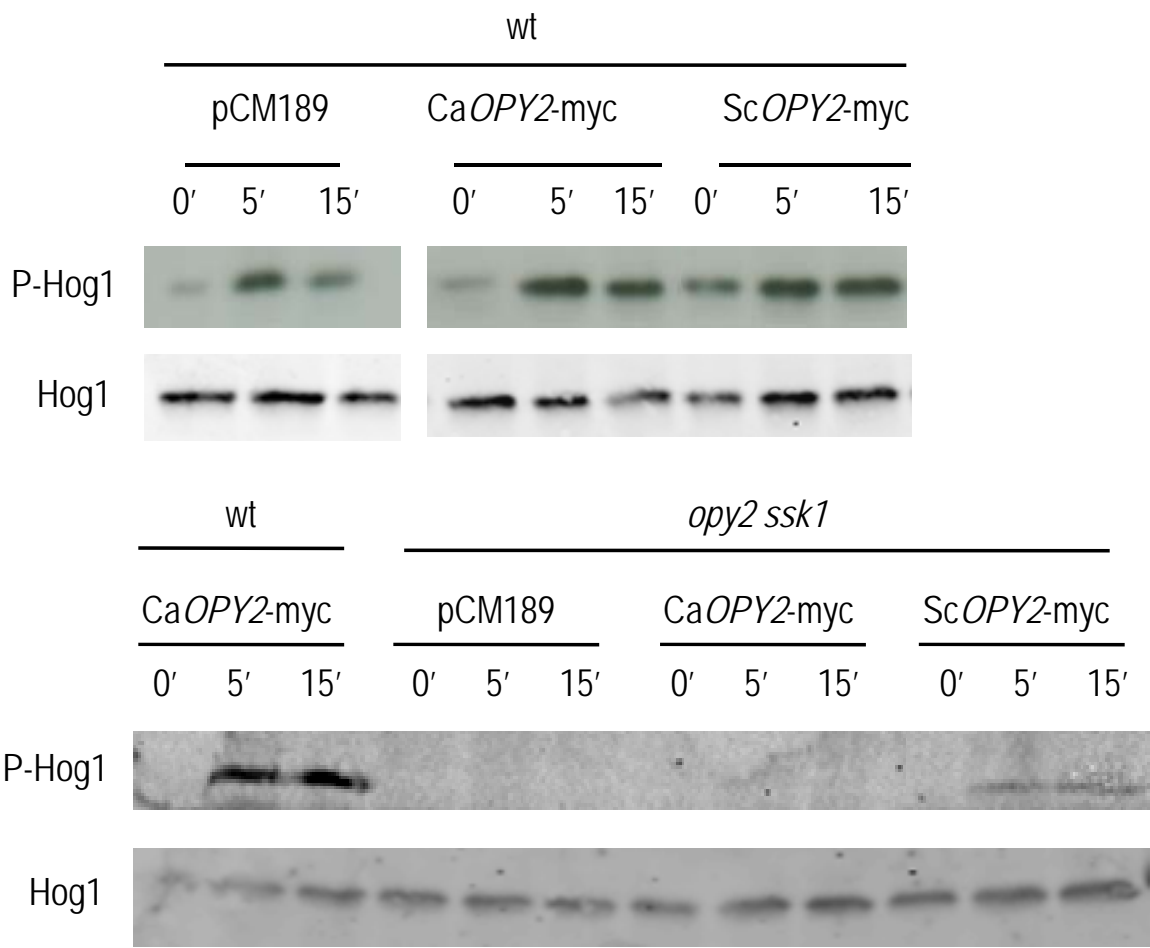

Figure 4 

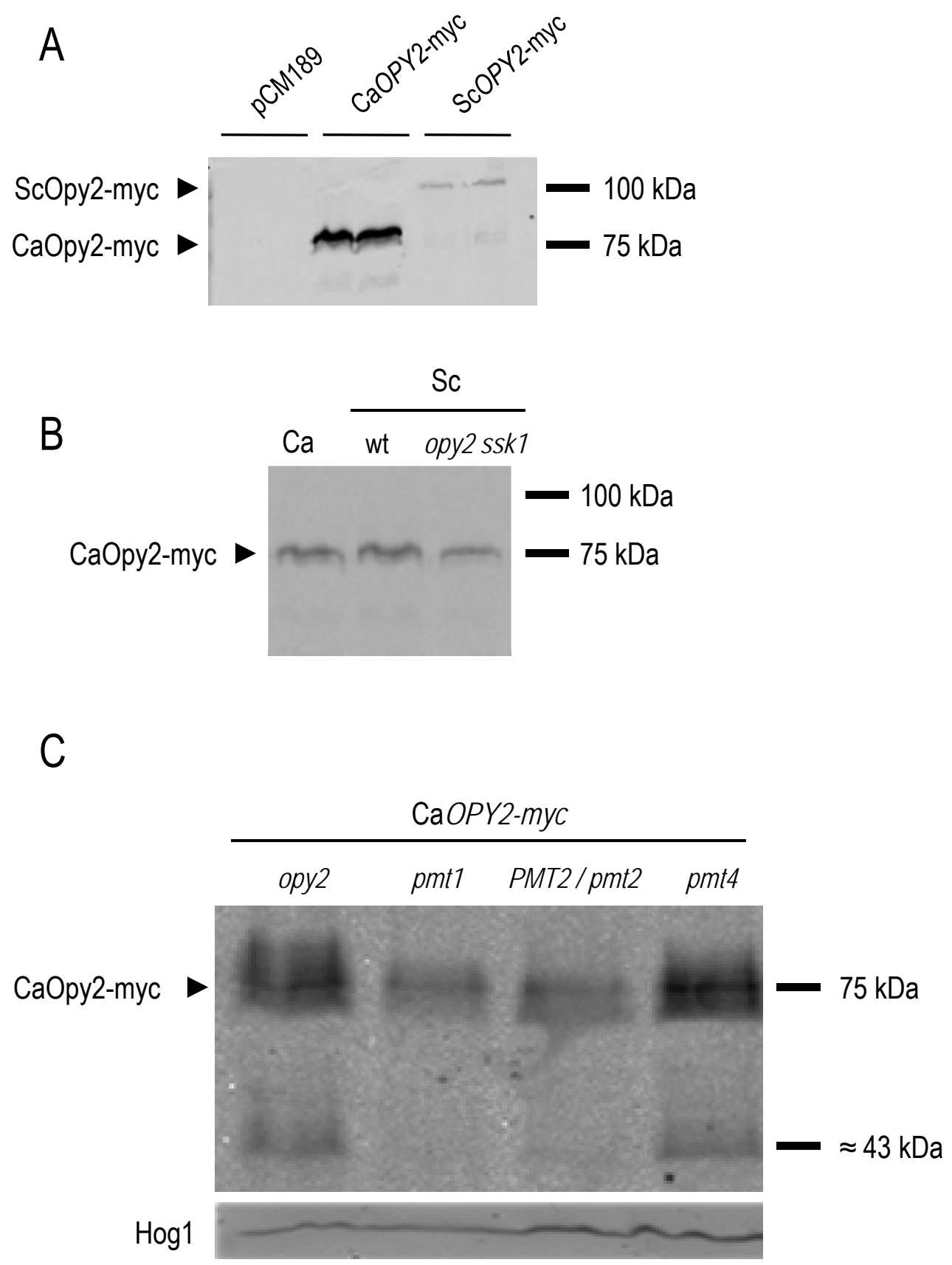

Figure 5 
A

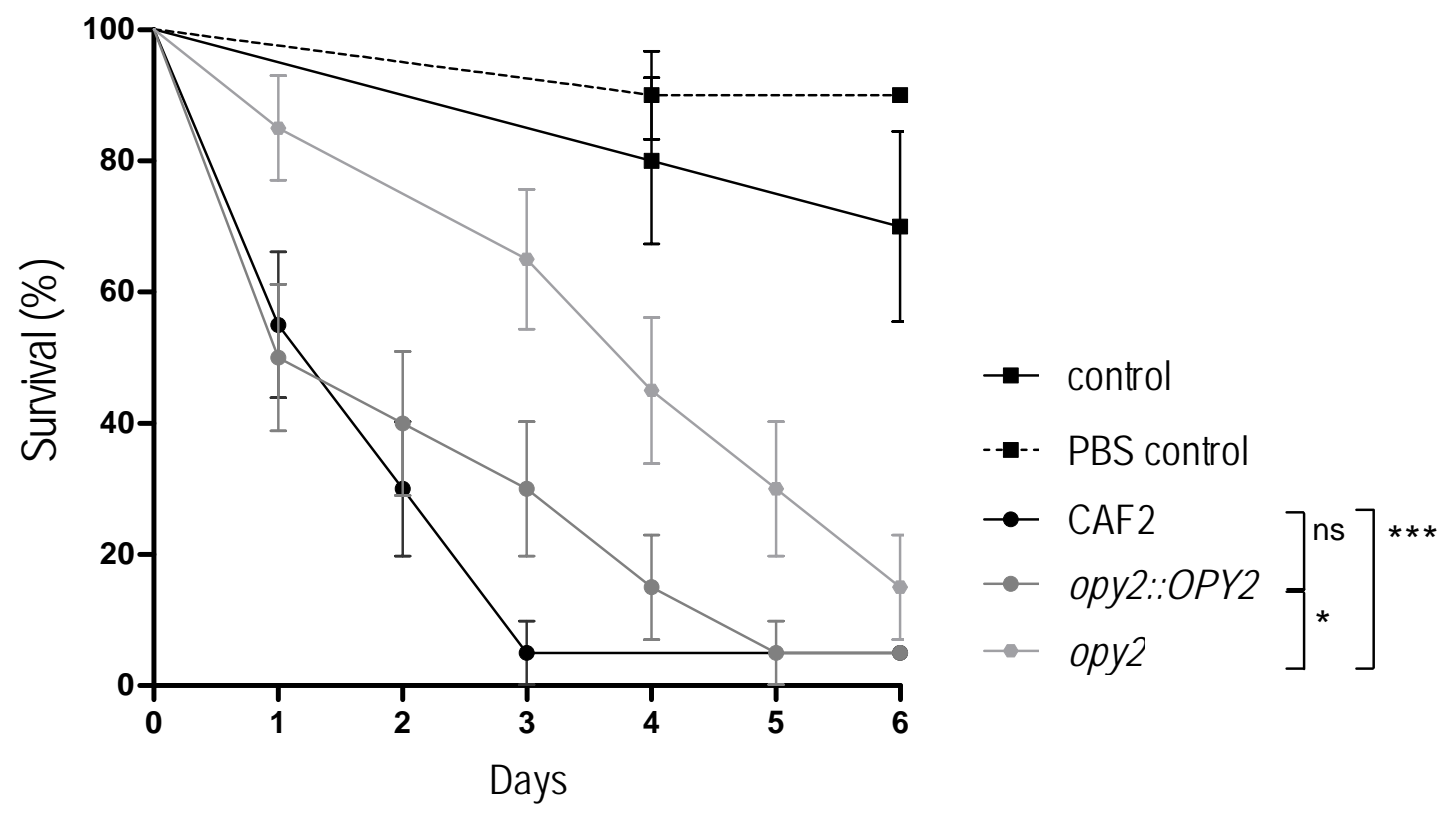

B

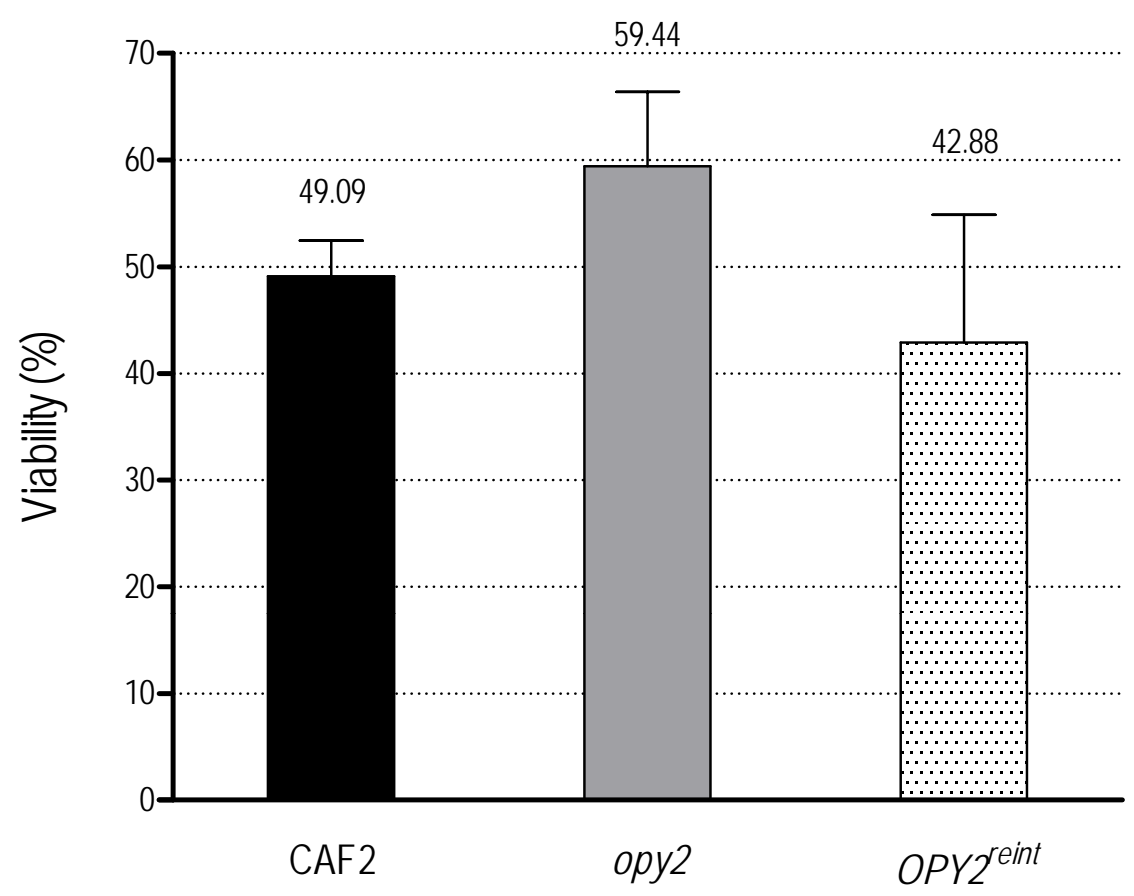

Figure 6 
A

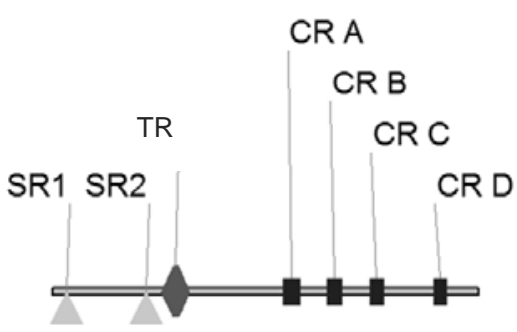

CaOpy2p

528 aa

ScOpy2p

361 aa

B
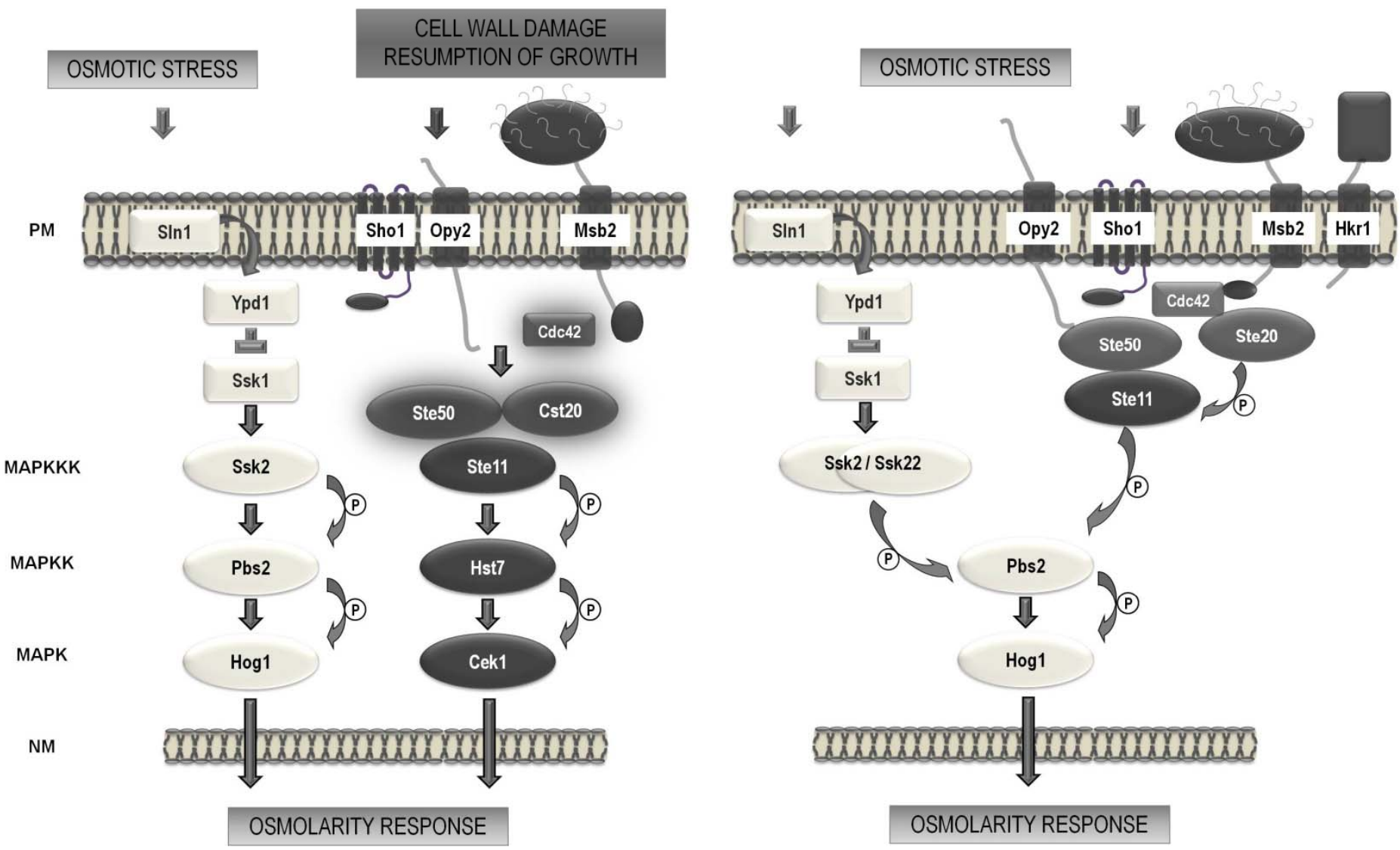

Figure 7 

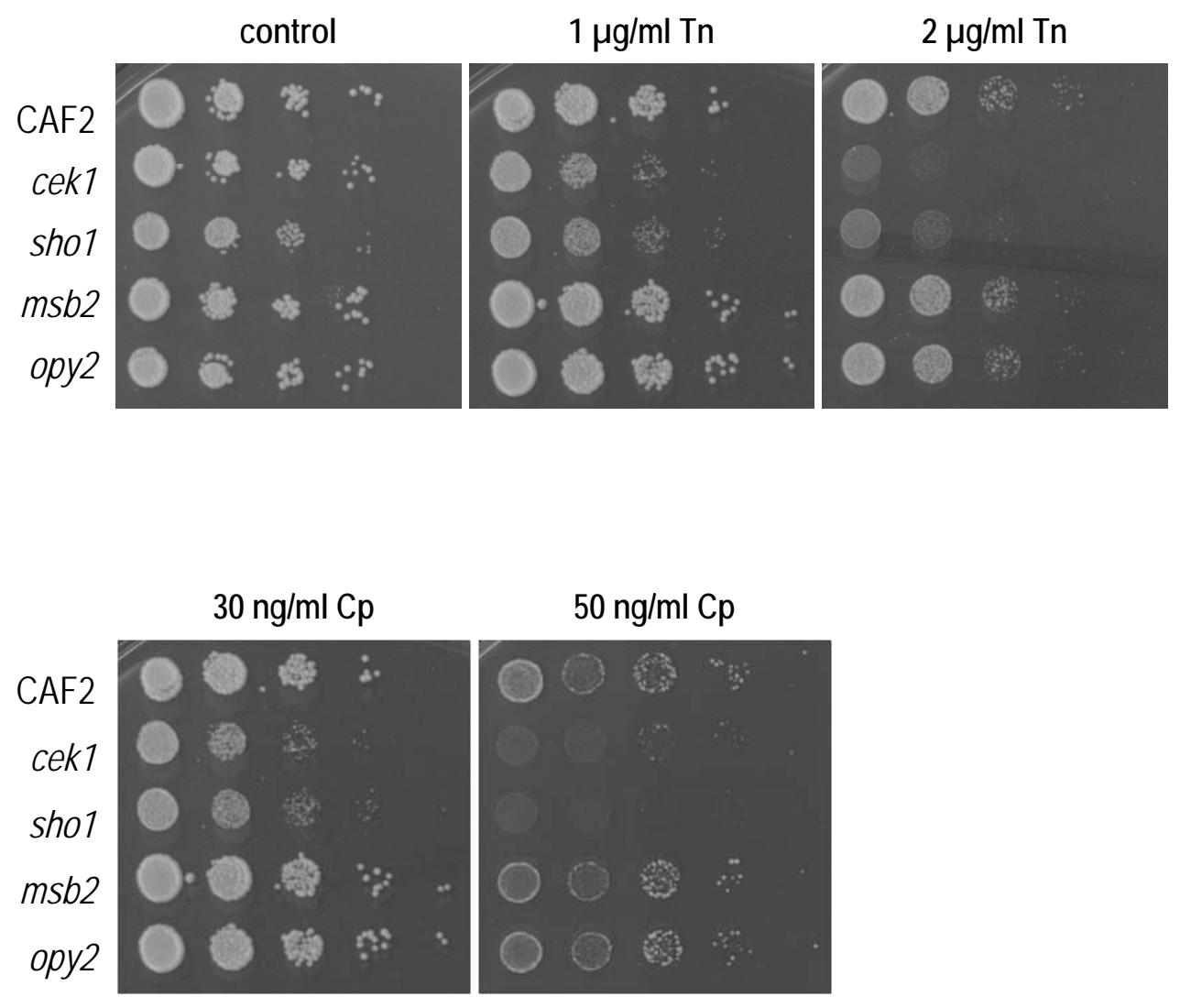


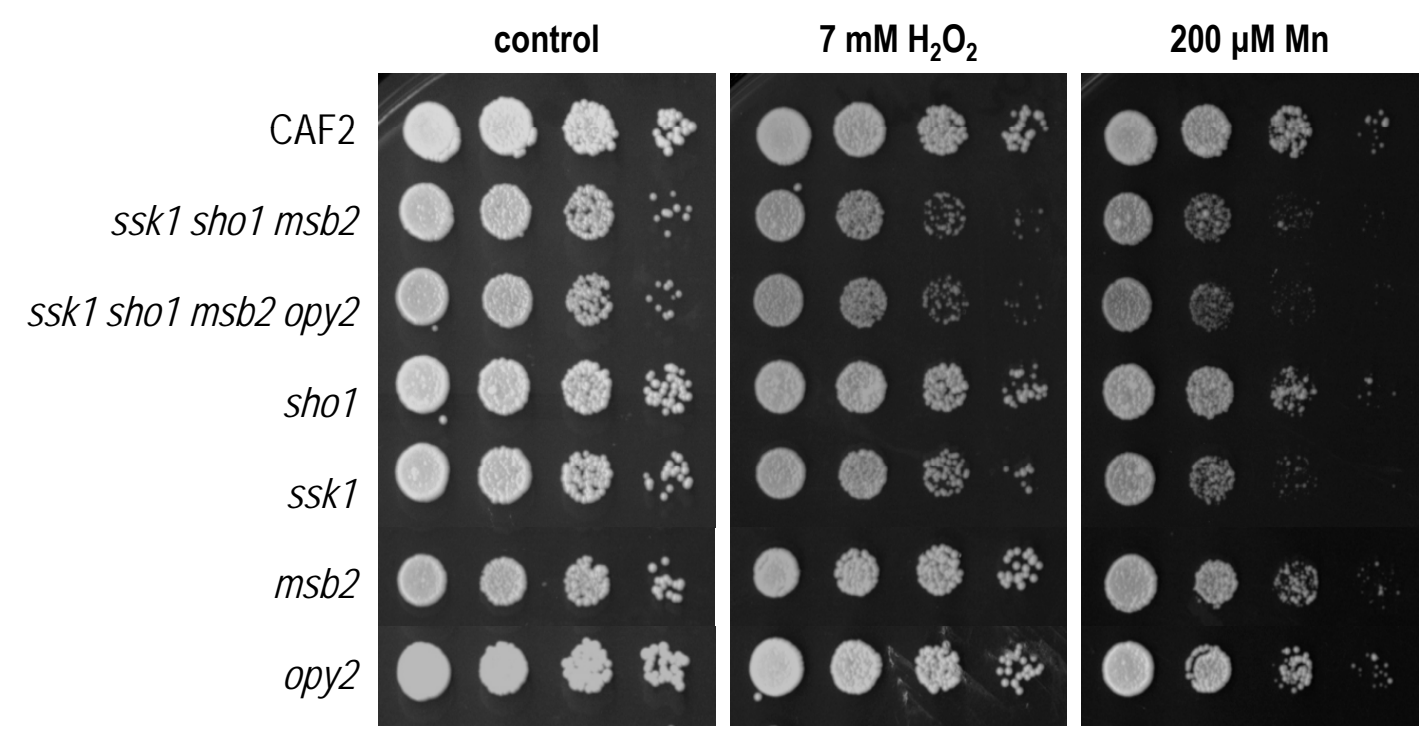

Supplementary 2 